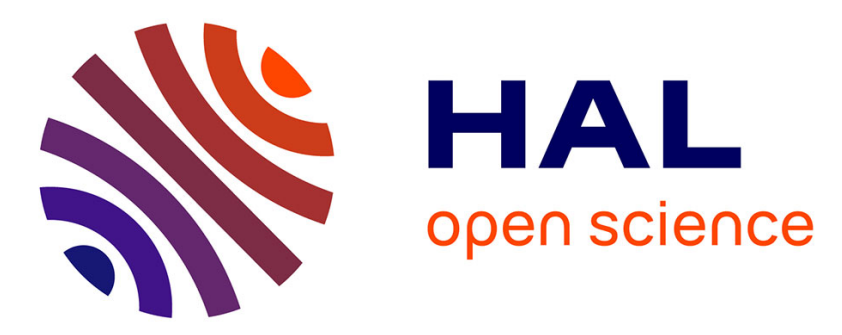

\title{
Statistical radiative modeling of a porous medium with semi transparent and transparent phases: Application to a felt of overlapping fibres
}

\author{
Yann Dauvois, Denis Rochais, Franck Enguehard, Jean Taine
}

\section{- To cite this version:}

Yann Dauvois, Denis Rochais, Franck Enguehard, Jean Taine. Statistical radiative modeling of a porous medium with semi transparent and transparent phases: Application to a felt of overlapping fibres. International Journal of Heat and Mass Transfer, 2017, 106, pp.601-618. 10.1016/j.ijheatmasstransfer.2016.09.035 . hal-01513610

HAL Id: hal-01513610

https://hal-centralesupelec.archives-ouvertes.fr/hal-01513610

Submitted on 25 Apr 2017

HAL is a multi-disciplinary open access archive for the deposit and dissemination of scientific research documents, whether they are published or not. The documents may come from teaching and research institutions in France or abroad, or from public or private research centers.
L'archive ouverte pluridisciplinaire HAL, est destinée au dépôt et à la diffusion de documents scientifiques de niveau recherche, publiés ou non, émanant des établissements d'enseignement et de recherche français ou étrangers, des laboratoires publics ou privés. 


\title{
Statistical radiative modeling of a porous medium with semi transparent and transparent phases: Application to a felt of overlapping fibres.
}

\author{
Yann Dauvois ${ }^{\mathrm{a}, \mathrm{b}}$, Denis Rochais ${ }^{\mathrm{b}}$, Franck Enguehard $^{\mathrm{a}}$, Jean Taine $^{\mathrm{a}}$ \\ ${ }^{a}$ CNRS, UPR 288, Laboratoire d'Énergétique Moléculaire et Macroscopique, Combustion (EM2C), \\ CentraleSupelec, Bâtiment Péclet, 92295 Châtenay-Malabry, France. \\ ${ }^{b}$ CEA, DAM, Le Ripault, BP16, 37260 Monts, France.
}

\begin{abstract}
A general statistical model of characterisation of the radiative properties of homogenised phases has been developed for a porous medium with a semi transparent absorbing phase a and a transparent one b, characterised by general interfacial reflection and transmission laws. For non Beerian homogenised phases, it is based on successive sets of radiative statistical functions: Extinction cumulative distribution functions, scattering cumulative probabilities and general phase functions ab initio determined by a Monte Carlo approach, only from morphological data and interfacial reflection and transmission laws in the last case. Specific sets are associated with isotropic and uniform volume emission by a and with the successive internal and external scattering events within a and b, the emission or scattering source terms of which have been weighted by spatial distribution functions. For a Beerian homogenised phase, a unique set of radiative statistical functions has been determined from random isotropic volume source points.

Two Generalised Radiative Transfer Equations (GRTEs), coupled by external scattering source terms are then expressed only vs the radiative statistical functions. It is shown that a radiative Fourier's model, based on radiative conductivity tensors, is not valid for a medium made of a semi transparent phase and a transparent one, if the particular case for which the semi transparent phase becomes opaque and the trivial case of a quasi isothermal medium are excepted.
\end{abstract}


The previous models are applied to a felt of fibres for insulation of high temperature systems. The radiative power field in radiation steady state within a felt of fibres enclosed between parallel opaque walls has been determined by solving the coupled GRTEs by a Monte Carlo method, for different values of the transverse optical thickness of a fibre. The temperature field within the felt has also been determined for two temperatures imposed at the boundaries and for imposed flux and temperature. Finally the optimal conditions of insulation have been determined for a case such that usual conduction can be neglected compared to radiation.

Keywords: semi transparent and transparent phases, statistical radiative properties model, non Beerian effective prperties, ab intio determined scattering phase functions, coupled Generalised Radiative Transfer Equations, fibrous medium.

\section{Nomenclature}

\section{Latin symbols}

n Normal unit vector towards the (semi) transparent phase

r Coordinates of a current point

$\mathbf{u} \quad$ unit vector of the current direction

$\mathbf{q} \quad$ Flux $\left(W \cdot m^{-2}\right)$

$B \quad$ Generalised extinction coefficient

$f \quad$ Distribution function

$G \quad$ Radiative cumulative distribution function

I Radiative intensity

$k^{R} \quad$ Radiative conductivity

M Volume source point 
$M^{\prime} \quad$ Extinction point

$N \quad$ Interfacial source point

$n \quad$ Refractive index

$P \quad$ Cumulative probability

$p \quad$ Phase function

$S \quad$ Radiative source term

$s, s^{\prime} \quad$ Curvilinear abscissas along a ray

T Temperature

V Volume

$z \quad$ Axis of the bed $f$ fibers

D Medium length

d Cylinder diameter

L Cylinder length

\section{Greek symbols}

$\beta \quad$ Extinction coefficient

$\kappa \quad$ Absorption coefficient

$\nu \quad$ Frequency

$\rho^{\prime \prime} \quad$ Bidirectional reflectivity

$\tau^{\prime \prime} \quad$ Bidirectional transmissivity

$\delta \quad$ Kronecker symbol 
$\Omega \quad$ Solid angle

$\Phi \quad$ Azimutal angle

$\Pi \quad$ Volume fraction or porosity

$\Sigma \quad$ Interfacial area

$\Sigma_{c c}$ or $\Sigma_{c d}$ Generalised scattering coefficient

$\theta \quad$ Angle related to the $z$ axis

\section{Subscripts}

$\begin{array}{ll}-a & \text { Absorption } \\ -{ }_{c} & \text { Phase a or b } \\ -{ }_{d} & \text { Another phase a or b } \\ -{ }_{e x t} & \text { Extinction } \\ -{ }_{e} \quad \text { Emission } & \\ -{ }_{H} & \text { Successive set of internal and external scattering events } \\ - & \quad \text { Scattering }\end{array}$

\section{Superscripts}

${ }^{(n)} \quad n^{\text {th }}$ set of scattering events

$-^{\circ}$ At equilibrium

${ }^{B} \quad$ Related to a Beerian phase

${ }^{S} \quad$ Related to an interfacial source

\section{Others}




\section{GRTE Generalised Radiative Transfer Equation}

RTE Radiative Transfer Equation

STT Medium with Semi Transparent and Transparent phases

\section{Introduction}

An accurate modeling of radiative transfer is required in applications involving heat transfer in a porous medium at high temperature. The radiative properties of the different phases have to be characterised separately in order to be able to account for coupling with other heat transfer modes, generally conduction in a solid phase and conduction or convection in a fluid phase. As radiative transfer cannot be in practice determined at local scale, effective properties of every phase have to be determined.

The methods of parameter identification with many variants based on experiments or numerical transfer simulation (see a detailed review in Ref.[1]) are the most popular methods of characterisation of these radiative effective properties. If they are relevant for media characterised by an exponential extinction law (Beerian media), their limitations for non Beerian media have been discussed elsewhere[2]. Moreover, these methods do not easily allow every phase to be separately characterised.

In the general case, the radiative effective properties of a phase of a porous medium, which is often statistically non homogeneous and anisotropic, are non Beerian. The conditions of validity of the Beer's law for characterising these radiative effective properties have been discussed in a recent paper [3]. The law is, in particular, valid if one of the following conditions is fulfilled:

i) When the phase is statistically homogeneous at all the spatial scales considered in the application; It can be non homogeneous at scales such that the medium is optically thin;

ii) When the interfaces of the porous medium present some special symmetries: For instance outside of overlapping spheres or cylinders;

iii) At spatial scales larger than $\delta$, length such that the medium is optically thick. In this popular case, extinction, scattering and absorption coefficients can be defined 
and a radiative conductivity tensor can be introduced (radiative Fourier's law).

A radiative model which can be applied to non Beerian media has been initiated by Consalvi et al.[4] and developed by Lipinski et al.[5]. This approach is similar to the volume averaging method of Whitaker and Quintard[6, 7], for obtaining effective properties of a porous medium in the case of small perturbations of the field within a representative volume element.

The development of $\mathrm{X}$ and $\gamma$ tomography techniques has led in the last decades to an accurate knowledge of the morphology of porous media. On the other hand the morphology of material models, sets of overlapping or non overlapping spheres or cylinders for instance, is exactly known. In these conditions, the increasing power of the statistical Monte Carlo methods has allowed the radiative properties of a non Beerian homogenised phase to be exhaustively characterised by radiative statistical functions instead of extinction, absorption and scattering coefficients, valid for a Beerian medium. The principles of the method have been defined by Tancrez and Taine[8], for models of foams. A non Beerian homogenised phase is characterised by an extinction cumulative distribution function, a scattering (or absorption) cumulative probability and a general scattering phase function a priori depending on both the incidence and scattering directions. In this first work and in following ones, for instance in Refs.[9-15], the Beerian assumption has been validated for statistically isotropic and homogeneous media.

An original transfer model for a non Beerian homogenised phase, directly based on a Generalised Radiative Transfer Equation (GRTE) involving the radiative statistical functions, has then been developed[16] for a medium with an opaque and a transparent phase, both in the general case and when a radiative Fourier's law is valid. The physical bases of the GRTE have been improved in a recent paper[3]. This model has been applied, under the Fourier's assumption, to ordered sets of intact or degraded diffuse opaque parallel cylinders within a transparent or a semi transparent medium[14]. This porous medium, modeling a nuclear core, is strongly anisotropic but homogeneous at large scale. In a recent work[17], the GRTE has been applied to a statistically strongly non homogeneous porous medium: A set of non overlapping 
diffuse opaque spheres at the vicinity of a wall within a transparent medium.

A key feature of a non Beerian phase is that the knowledge of the incident intensity field at a given point $M$ is not sufficient for allowing extinction by absorption, external scattering or internal scattering at this point $M$ to be determined $[3,16]$ : The history of all radiations issued from all source points which contribute to the intensity field at $M$ has to be accounted for (memory effect). This phenomenon makes the model much more complex, in particular within a medium with transparent and semi transparent phases characterised by general (non diffuse) interfacial reflection and transmission laws: It is then necessary to account for all the previous paths involving different sets of effective internal and external scattering, associated with interfacial reflection and transmission phenomena[3]. On the contrary, under the particular assumption of an interfacial diffuse reflection or transmission law, the knowledge of the local intensity is sufficient for characterising internal or external scattering, as the directional contributions to these phenomena are uniformly jammed[3].

Fibrous media like felts are commonly used as insulating materials at high temperatures and can be encountered in applications such as nuclear reactors, combustion chambers, coatings for aeronautics and aerospace, etc. Radiative transfer in fibrous media has often been studied for opaque fibres immersed in a transparent phase. After a global homogenisation, this type of medium has generally been globally represented by a Beerian semi transparent medium. Radiative transfer is then often solved by a finite volume method (FVM) or a discrete ordinates method (DOM) [18-22]. Kamdem and Baillis [23] have replaced an anisotropic fibrous medium by an equivalent homogeneous isotropic medium and solved the radiation problem by classical techniques.

A common difficulty is the determination of the phase function of an anisotropic fibrous medium. Most studies have used a Henyey-Greenstein phase function, simply depending on the scattering angle and characterised by the asymmetry factor. Kamdem and Baillis [24] also have proposed a generalised Henyey-Greenstein phase function for an anisotropic medium by using a directional asymmetry factor. Lee [25] has proposed a general expression of the phase function for an oriented infi- 
nite cylinder and generalized the expression for fibrous media, but in this approach the interaction between fibres are neglected. Boulet and al. [26] have shown that these methods do not satisfy the energy conservation with anisotropic scattering. They have proposed a renormalisation of the phase function, but this renormalisation modifies its general shape and consequently the solution of the radiative transfer problem. Mishchenko et al. [27] multiply the Gaussian weights of the DOM by correction factors which affect the forward-scattering and ensure energy conservation, but this technique modifies the asymmetry factor for any direction. Hunter et al. [28] improve the previous technique by normalizing the backward-scattering term and the forward-scattering term of the phase function separately for specular and diffuse reflection and transmission laws. They conserve both scattered energy and asymmetry factor.

Section 2 deals with the radiation model for an assumed non Beerian porous medium with a semi transparent absorbing phase and a transparent one, characterised by general interfacial reflection-transmission laws: This model is for instance required in the case of Fresnel's interfacial laws. The medium is statistically anisotropic, but homogeneous at large scale. It corresponds, for instance, to another common type of felts.

An originality of the work is to apply the statistical approach earlier evoked for exhaustively characterising the extinction cumulative distribution functions and the scattering (or absorption) cumulative probabilities. A particular attention is also brought to the direct ab initio determination of all phase functions associated with all the sets of successive internal and external scattering events previously mentioned. This model is only based on the knowledge of the morphological properties of the medium, the refractive indices of the two phases and the absorption coefficient of the semi transparent phase. In the general case, the radiative transfer is based on two coupled Generalised Radiative Transfer Equations (GRTEs). The validity conditions of radiative Fourier's laws and radiative conductivity tensors, which require strong conditions, are discussed.

The radiative properties of a felt of semi transparent finite overlapping cylindrical fi- 
bres within a transparent medium are characterised by the previous method in Sec.3. Some applications to radiative transfer are developed in Sec.4.

\section{Radiation models}

\subsection{Statistical model of radiative properties}

The study deals with statistically homogeneous but strongly anisotropic media with an absorbing but non scattering phase a of volume fraction $\Pi_{a}$, absorption coefficient $\kappa_{\nu a}$ and refractive index $n_{\nu a}$ and a transparent phase b of volume fraction $\Pi_{b}$ and refractive index $n_{\nu b}$. All these thermophysical quantities are assumed uniform. Interfacial reflection and transmission at local scales are characterised by bidirectional reflectivity and transmissivity, given for instance by the Fresnel's laws for optically smooth interfaces.

The considered spatial scales are assumed to be larger than the typical radiation wavelengths: Consequently, diffraction effects are neglected and the radiation propagation is considered along straight lines within a phase.

By generalising the approach of Refs.[3, 5, 8, 9, 14, 15, 17, 29], the radiative properties of a priori non Beerian homogenised phases are accurately characterised by radiative statistical functions, defined along any propagation axis of direction $\mathbf{u}(\theta, \varphi)$ and coordinate $s$ : Extinction cumulative distribution function $G_{\text {ext } \nu}$, absorption or scattering cumulative probabilities, $P_{a \nu}$ or $P_{s c \nu}$ respectively, and general phase functions $p_{\nu}\left(\mathbf{u}_{1}, \mathbf{u}\right)$ a priori depending on both incidence and scattering unit vectors $\mathbf{u}_{1}$ and $\mathbf{u}$, respectively. Note that reflection within a real phase c (a or b) becomes internal scattering, characterised by $P_{s c \nu c c}$ and $p_{\nu c c}$ in the corresponding homogenised phase, and that transmission from a real phase $\mathrm{c}$ to another real phase d becomes external scattering characterised by $P_{s c \nu c d}$ and $p_{\nu c d}$ after homogenisation.

The radiative statistical functions are determined in the real medium by a statistical Monte Carlo method. A huge number of shots from emission and scattering source points is used for modeling extinction by absorption and scattering and modeling scattering source terms. This approach is summarised in Sec.2.1.4. The determined statistical functions are then used in the homogenised phases. Any homogenised phase is present at any point of the medium, more precisely it is charac- 
terised at this point by a presence probability, which is equal to its volume fraction $\Pi[16]$. For instance, emission by the absorbing phase a occurs at any point $M$ and is characterised by $\Pi_{a} \kappa_{\nu a} n_{\nu a}^{2} I_{\nu}^{\circ}\left[T_{a}(\mathbf{r})\right]$, where $\Pi_{a}$ accounts for the presence probability of a.

In a non Beerian homogenised phase, the radiation intensity can be determined at any point $M(\mathbf{r})$ in a given direction $\mathbf{u}_{1}$, but it does not allow its variation between two close points $M(\mathbf{r})$ and $M^{\prime}(\mathbf{r}+\mathrm{d} \mathbf{r})$ to be determined[3, 16]. It is a memory effect of the structure of this medium which is non homogeneous at small scale. Radiation transfer is then directly based on the previous radiative statistical functions.

Within the popular assumption diffuse reflection and transmission laws at the interfaces, the only knowledge of the global intensity field at any point $M$ of the homogenised phase is sufficient for determining the scattered intensity field at $M$, by using the corresponding phase function. This phase function is then simply determined in the real medium by summing all the contributions associated with the local reflection and transmission laws. But, in the present case, difficulties of modeling are due to the non diffuse interfacial reflection and transmission laws. In these conditions, as developed in the following, the phase function cannot be determined as simply as previously. In fact, different phase functions are associated with the successive sets of scattering events, corresponding to reflection and transmission phenomena within the real medium. The knowledge of the spatial distribution function of the incidence directions at any point of the real medium is then necessary for any set of scattering events.

In parallel, the knowledge of the spatial distribution function of scattered directions corresponding to a type of scattering events at any point of the real medium, is also necessary for determining the different extinction cumulative distribution functions and consequently scattering cumulative probabilities also associated with these events as source terms.

The different types of extinction cumulative distribution functions, scattering and absorption cumulative probabilities and finally phase functions are defined and expressed in this Section. 


\subsubsection{Extinction}

The extinction cumulative distribution function $G_{\text {ext } \nu a}\left(\mathbf{u}, s^{\prime}-s\right)$ associated with isotropic volume emission by $a$ is, in the homogenised phase, the probability that a radiation emitted in the direction $\mathbf{u}$ at the point $M(\mathbf{r})$, of abscissa $s$ along the axis of direction $\mathbf{u}$, is absorbed before a point $M^{\prime}\left(\mathbf{r}^{\prime}\right)$, of abscissa $s^{\prime}$, within the phase a or internally or externally scattered at an interface with the transparent phase between $s$ and $s^{\prime}$. $G_{\text {ext } \nu a}\left(\mathbf{u}, s^{\prime}-s\right)$ is also equal to $1-\tau_{\nu a}\left(\mathbf{u}, s^{\prime}-s\right)$, where $\tau_{\nu a}$ is the transmissivity from $s$ to $s^{\prime}$ in the direction $\mathbf{u}$.

As extinction by absorption within the real Beerian medium a and extinction by interfacial scattering are statistically independent, the transmissivity $\tau_{\nu a}\left(\mathbf{u}, s^{\prime}-s\right)$ is the product of the partial transmissivities associated with these two phenomena and $G_{\text {ext } \nu a}\left(\mathbf{u}, s^{\prime}-s\right)$ also writes

$$
G_{e x t \nu a}\left(\mathbf{u}, s^{\prime}-s\right)=1-\exp \left[-\kappa_{\nu a}\left(s^{\prime}-s\right)\right]\left[1-P_{s c a}^{t}\left(\mathbf{u}, s^{\prime}-s\right)\right]
$$

where $P_{s c ~}^{t}$ is the scattering cumulative probability associated with an assumed transparent phase a. As extinction by scattering is independent of the radiation frequency, $P_{s c a}^{t}$ simply is a geometrical quantity. Note that $P_{s c a}^{t}$ can also be considered as the extinction cumulative distribution function $G_{\text {ext } a}^{t}$ of the assumed transparent phase a. Finally, $P_{s c ~ a}^{t}$ is the cumulative distribution function of the lengths $s^{\prime}-s$ in the direction $\mathbf{u}$ between any volume point $M(s)$ of a and the corresponding interfacial impact point $E\left(s_{0}\right)$ which writes

$$
P_{s c a}^{t}\left(\mathbf{u}, s^{\prime}-s\right)=\frac{1}{\Pi_{a} V} \frac{1}{\delta \Omega(\mathbf{u})} \int_{0}^{s^{\prime}-s} \int_{\Pi_{a} V} \int_{\delta \Omega(\mathbf{u})} \delta\left(v-\left[s_{0}\left(\mathbf{u}^{\prime}, \mathbf{r}\right)-s\left(\mathbf{u}^{\prime}, \mathbf{r}\right)\right]\right) \mathrm{d} \Omega\left(\mathbf{u}^{\prime}\right) \mathrm{d} \mathbf{r} \mathrm{d} v
$$

where the summations are carried out, within the assumed real transparent phase a, over all directions $\mathbf{u}^{\prime}$ of an elementary solid angle $\delta \Omega(\mathbf{u})$ around the direction $\mathbf{u}$, over all points $M(\mathbf{r})$ of the volume $\Pi_{a} V$ of the phase a and over the distance $v=s "-s$ between $P\left(\mathbf{r}^{\prime \prime}\right)$, current point of the axis $\mathbf{u}^{\prime}$ and $M(\mathbf{r})$, the source point over this axis; $s\left(\mathbf{u}^{\prime}, \mathbf{r}\right)$, and $s_{0}\left(\mathbf{u}^{\prime}, \mathbf{r}\right)$ are the abscissas of the source and extinction points $M$ and $E$, respectively; $\delta$ is the Dirac distribution. 


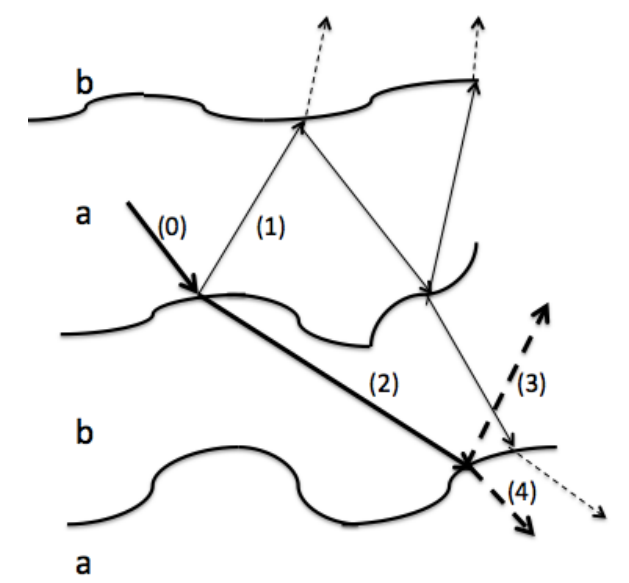

Figure 1: First successive sets of internal and external scattering events.

Different extinction cumulative distribution functions of the type $G_{\text {ext } \nu}^{S(n)}$ characterising the phases a and b are also associated with the source terms constituted by the successive sets of internal and external scattering events (see Fig.1); The superscript ${ }^{S}$ means that these quantities are related to interfacial sources. The interfacial source points of the $n^{\text {th }}$ set of scattering events belong to the part $\Sigma^{(n)}$ of the interface area $\Sigma$ which has been illuminated by the rays of the previous $(n-1)^{t h}$ set of scattering events. More precisely, these source points $N\left(\mathbf{r}_{n}\right)$ of the surface $\Sigma^{(n)}$ are characterised in the real medium by the spatial distribution function $f_{\mathbf{u}}^{S{ }^{(n)}}\left(\mathbf{r}_{n}\right)$. A specific extinction cumulative distribution function $G_{e x t \nu}^{S(n)}$ is then associated with this $n^{\text {th }}$ set of scattering source terms in the direction $\mathbf{u}$ within a homogenised phase. The distribution function $f_{\mathbf{u}}^{S{ }^{(n)}}\left(\mathbf{r}_{n}\right)$ depends on all the history of the $n-1$ previous sets of scattering events, i.e. on the successive phenomena of internal and external scattering and partial absorption of a radiation initially emitted within the phase a. It is determined with a Monte Carlo statistical approach, the principles of which are defined in Sec.2.1.4.

A given spatial distribution function of the scattered direction $\mathbf{u}$ and the corresponding extinction cumulative distribution function are noted $f_{\mathbf{u} \text { acHde }}^{S(n)}(\mathbf{r})$ and $G_{\text {ext }}^{S(n)}$ acHde . The symbolic notation acHde defines the history of this set, which is always issued from isotropic emission within the phase a: i) c designates the propagation medium 
of the first set of scattering events: $a$ in case of internal scattering within $a, b$ in case of external scattering from a to b; ii) The condensed symbol $H$ represents successive sets of internal and external scattering events; iii) $d$ specifies the phase which is source of the considered $n^{\text {th }}$ set of scattering events; iV) $e$ defines the propagation phase of this set. For instance, aabbaa corresponds to volume emission within a, followed by internal scattering within a, external scattering towards b, internal scattering within b, external scattering towards a, internal scattering within a where the ray is finally extinguished by volume absorption or interfacial scattering.

In these conditions, $G_{\text {ext } \nu \text { acHde }}^{S(n)}$ is given by an expression similar to Eq.1 vs $P_{\text {sc } t S a c H d e}^{t S(n)}$, total scattering cumulative probability in an assumed transparent phase, which writes

$$
\begin{aligned}
& P_{\text {sc vacHde }}^{t S(n)}\left(\mathbf{u}, s^{\prime}-s\right)= \\
& \frac{1}{\delta \Omega_{e}(\mathbf{u})} \int_{0}^{s^{\prime}-s} \int_{\Sigma^{(n)} / \delta V / \mathbf{u} \cdot \mathbf{n}_{\mathbf{e}}\left(\mathbf{r}_{n}\right)>0} \int_{\delta \Omega_{e}(\mathbf{u})} f_{\mathbf{u}^{\prime} a c H d e}^{S(n)}\left(\mathbf{r}_{n}\right) \delta\left(v-\left[s_{0}\left(\mathbf{u}^{\prime}, \mathbf{r}_{n}\right)-s\left(\mathbf{u}^{\prime}, \mathbf{r}_{n}\right)\right]\right) \mathrm{d} \Omega_{e}\left(\mathbf{u}^{\prime}\right) \mathrm{d} \mathbf{r}_{n} \mathrm{~d} v .
\end{aligned}
$$

where the summations are carried out: i) Over the directions $\mathbf{u}^{\prime}$ of an elementary solid angle $\delta \Omega_{e}(\mathbf{u})$ of the final medium e; ii) Over all the points $M\left(\mathbf{r}_{n}\right)$ of abscissa $s\left(\mathbf{u}^{\prime}, \mathbf{r}_{n}\right)$ of $\Sigma^{(n)}$ such that $\mathbf{u} . \mathbf{n}_{e}\left(\mathbf{r}_{n}\right)>0$, where $\mathbf{n}_{e}$ is the unit vector normal to $\Sigma^{(n)}$ oriented towards the phase e; iii) Over the distance $v=s "-s$ between the source point $M(s)$ and the current point $P$ of the axis $\mathbf{u}^{\prime} ; s_{0}\left(\mathbf{u}, \mathbf{r}_{n}\right)$ is the abscissa of a current extinction point $E$.

If a homogenised phase is Beerian, the previous model is drastically simplified[3]. Indeed, in this case the knowledge of the incidence intensity field at a point allows the scattered intensity field at the same point to be directly determined. sWhatever the interfacial reflection and transmission laws, a unique extinction cumulative distribution function $G_{\text {ext } \nu c}\left(\mathbf{u}, s^{\prime}-s\right)$ characterises this phase. It is practically determined by equations identical to Eqs.1 and 2, i.e. by using isotropic volume source points as shown by Dauvois et al.[3].

\subsubsection{Scattering and absorption}

The absorption, internal scattering and external scattering cumulative probabilities $P_{a \nu a}\left(\mathbf{u}, s^{\prime}-s\right), P_{s c \nu a a}\left(\mathbf{u}, s^{\prime}-s\right)$ and $P_{s c \nu a b}\left(\mathbf{u}, s^{\prime}-s\right)$ associated with isotropic 
volume emission by a are respectively, in the homogenised phase, the probabilities that a radiation emitted in the direction $\mathbf{u}$, at the point $M(s)$ is absorbed within the phase a, internally scattered or externally scattered at an interface with the transparent phase, between $s$ and $s^{\prime}$. These quantities are linked by

$$
G_{\text {ext } \nu a}=P_{a \nu a}+P_{s c \nu a a}+P_{s c \nu a b}
$$

The previous quantities do not account for scattering or absorption of radiation belonging to the successive sets of scattering events.

The extinction cumulative distribution function $G_{\text {ext } \nu \text { acHde }}^{S(n)}$ associated with the $n^{t h}$ set of successive scattering events in the direction $\mathbf{u}$ within a homogenised phase similarly is the sum of the corresponding internal and external scattering cumulative probabilities $P_{s c \nu \text { acHdee }}^{S(n)}\left(\mathbf{u}, s^{\prime}-s\right)$ and $P_{s c \nu \text { acHded }}^{S(n)}\left(\mathbf{u}, s^{\prime}-s\right)$ and possibly absorption cumulative probabilities $P_{a \nu \text { acHde }}^{S(n)}\left(\mathbf{u}, s^{\prime}-s\right)$, i.e.

$$
\begin{aligned}
& G_{\text {ext } \operatorname{SacHda}}^{S(n)}\left(\mathbf{u}, s^{\prime}-s\right)=P_{s c \nu a c H d a a}^{S(n)}\left(\mathbf{u}, s^{\prime}-s\right)+P_{s c \nu a c H d a b}^{S(n)}\left(\mathbf{u}, s^{\prime}-s\right)+P_{a \nu a c H d a}^{S(n)}\left(\mathbf{u}, s^{\prime}-s\right) ; \\
& G_{\text {extacHdb}}^{S(n)}\left(\mathbf{u}, s^{\prime}-s\right)=P_{s c a c H d b}^{t S(n)}\left(\mathbf{u}, s^{\prime}-s\right)=P_{s c \nu a c H d b a}^{t S(n)}\left(\mathbf{u}, s^{\prime}-s\right)+P_{s c \nu a c H d b b}^{t S(n)}\left(\mathbf{u}, s^{\prime}-s\right) .
\end{aligned}
$$

The previous scattering and absorption cumulative probabilities are determined in parallel with $G_{\text {ext } \nu \text { acHde }}^{S(n)}\left(\mathbf{u}, s^{\prime}-s\right)$ in the statistical approach of Sec.2.1.4. They represent the probabilities that a radiation issued from the $n^{\text {th }}$ scattering source term in the direction $\mathbf{u}$, at the point $M(s)$ of the homogenised phase e, is internally or externally scattered at an interface with the other phase, or absorbed within the phase a, between $s$ and $s^{\prime}$.

\subsubsection{Phase functions}

A specific phase function is associated in this Section with any type of previously defined scattering cumulative probability.

For a Beerian homogenised phase, the knowledge of the intensity at a point $M$ allows the scattered intensity at the same point to be determined from the corresponding phase function. Indeed, such a phase is homogeneous at all the considered spatial 
scales and the intensity brings a complete information for determining the evolution of the radiation from $M$ to an arbitrary close point $M^{\prime}[3,16]$.

The expression of the phase function associated with internal scattering within a Beerian homogenised phase has been given in Ref.[8] for instance. The phase function associated with external scattering from a, assumed Beerian homogenised phase, to b writes

$$
\begin{aligned}
p_{\nu a b}^{B}\left(\mathbf{u}_{\mathbf{1}}, \mathbf{u}\right) \frac{\mathrm{d} \Omega_{b}}{4 \pi} & = \\
& \frac{\int_{\Pi_{a} V / \mathbf{u}_{1} \cdot \mathbf{n}_{a} \leq 0} \tau_{\nu a b}^{\prime \prime}\left[\mathbf{u}_{\mathbf{1}}, \mathbf{u}, \mathbf{n}_{a}\left(\mathbf{r}, \mathbf{u}_{1}\right)\right]\left[-\mathbf{u}_{\mathbf{1}} \cdot \mathbf{n}_{a}\left(\mathbf{r}, \mathbf{u}_{1}\right)\right] \mathrm{d} \mathbf{r} \quad \mathrm{d} \Omega_{b}}{\int_{0}^{4 \pi} \int_{\Pi_{a} V / \mathbf{u}_{1} . \mathbf{n}_{a} \leq 0} \tau_{\nu a b}^{\prime \prime}\left[\mathbf{u}_{\mathbf{1}}, \mathbf{u}^{\prime}, \mathbf{n}_{a}\left(\mathbf{r}, \mathbf{u}_{1}\right)\right]\left[-\mathbf{u}_{\mathbf{1}} \cdot \mathbf{n}_{a}\left(\mathbf{r}, \mathbf{u}_{1}\right)\right] \mathrm{d} \mathbf{r} \mathrm{d} \Omega_{b}^{\prime}} .
\end{aligned}
$$

where $\tau_{\nu a b}^{\prime \prime}\left[\mathbf{u}_{\mathbf{1}}, \mathbf{u}, \mathbf{n}_{a}\left(\mathbf{r}, \mathbf{u}_{1}\right)\right]$ is the bidirectional transmissivity, $\mathbf{u}_{1}$ and $\mathbf{u}$ the incidence and scattering unit vectors and $\mathbf{n}_{a}$ the local normal unit vector oriented towards a at a current interfacial point of the real medium.

On the contrary, for a non Beerian homogenised phase a with non diffuse interfaces, the knowledge of the intensity at a point is not sufficient for determining the scattered intensity at this point. The "memory effect" within a non Beerian homogenised phase has to be accounted for: The scattered intensity at a point $M$ depends on the spatial distribution of the initial source points and on possible absorption between the source points and $M$.

Two phase functions are associated with the extinction by scattering of the rays of the $n^{\text {th }}$ set of scattering events and correspond to the scattering cumulative probabilities $P_{s c \nu \text { acHdee }}^{S(n)}\left(\mathbf{u}, s^{\prime}-s\right)$ and $P_{s c \nu \text { acHded }}^{S(n)}\left(\mathbf{u}, s^{\prime}-s\right)$ previously defined: They are noted $p_{\nu \text { acHdee }}^{S(n)}\left(\mathbf{u}_{1}, \mathbf{u}\right)$ and $p_{\nu \text { acHded }}^{S(n)}\left(\mathbf{u}_{1}, \mathbf{u}\right)$. These phase functions not only depend on the reflection-transmision laws at the impact points $M_{n}$ but also on $f_{i \mathbf{u}_{1}}^{S(n-1)}\left(\mathbf{r}_{n-1}\right)$, the spatial distribution function of the initial source points $M_{n-1}\left(\mathbf{r}_{n-1}\right)$. These points belong to the part $\Sigma^{(n-1)}$ of the interface area within the considered porous medium volume $V$ which is illuminated by the $(n-2)^{t h}$ scattering events and are the origins of the different components of the radiation incident over the surface $\Sigma^{(n)}$ in the direction $\mathbf{u}_{1}$. The function $f_{i \mathbf{u}_{1}}^{S(n-1)}\left(\mathbf{r}_{n-1}\right)$ is determined along an optically thick distance $\delta\left(\mathbf{u}_{1}\right)$ along any considered direction $\mathbf{u}_{1}$. 
The two first phase functions $p_{\nu a a}^{S(0)}\left(\mathbf{u}_{1}, \mathbf{u}\right)$ and $p_{\nu a b}^{S(0)}\left(\mathbf{u}_{1}, \mathbf{u}\right)$ are associated with $P_{s c \nu a a}\left(\mathbf{u}_{1}\right)$ and $P_{s c \nu a b}\left(\mathbf{u}_{1}\right)$ respectively which correspond to isotropic emission volume source points $\mathbf{r}_{0}$, or the zero ${ }^{\text {th }}$ set of rays, within the non Beerian phase a (see Fig.1). The corresponding spatial distribution function of the initial source points associated with the incidence direction $\mathbf{u}_{1}$ is noted $f_{i}^{S(0)}\left(\mathbf{u}_{1}\right)$. Its determination requires a supplementary assumption: The phase a is isothermal along any optically thick distance $\delta\left(\mathbf{u}_{1}\right)$ in any incidence direction $\mathbf{u}_{1}$. Note that this assumption is only required for the determination of the phase function. Emission of radiation can be modeled for a non isothermal phase at any spatial scale. In these conditions, $f_{i \mathbf{u}_{1}}^{S(0)}\left(\mathbf{r}_{0}\right)$ writes

$$
f_{i \mathbf{u}_{1}}^{S(0)}\left(\mathbf{r}_{0}\right)=\frac{\exp \left(-\kappa_{\nu a}\left[s_{0}\left(\mathbf{u}_{1}, \mathbf{r}_{0}\right)-s\left(\mathbf{u}_{1}, \mathbf{r}_{0}\right)\right]\right)}{\int_{\Pi_{a} V} \exp \left(-\kappa_{\nu a}\left[s_{0}\left(\mathbf{u}_{1}, \mathbf{r}_{0}\right)-s\left(\mathbf{u}_{1}, \mathbf{r}_{0}\right)\right]\right) \mathrm{d} \mathbf{r}_{0}}
$$

where $s\left(\mathbf{u}_{1}, \mathbf{r}_{0}\right)$ is the abscissa of the current volume isotropic emission source point over the axis of direction $\mathbf{u}_{1}$ and $s_{0}\left(\mathbf{u}_{1}, \mathbf{r}_{0}\right)$ the abscissa of the corresponding impact point $N\left(\mathbf{u}_{1}, \mathbf{r}_{0}\right)$ through an assumed transparent phase a. The phase functions $p_{\nu a a}^{S(1)}\left(\mathbf{u}_{1}, \mathbf{u}\right)$ and $p_{\nu a b}^{S(2)}\left(\mathbf{u}_{1}, \mathbf{u}\right)$ finally depend both on the bidirectional reflectivity $\rho_{a a \nu}^{\prime \prime}\left[\mathbf{u}_{\mathbf{1}}, \mathbf{u}, \mathbf{n}_{a}\left(\mathbf{u}_{1}, \mathbf{r}_{0}\right)\right]$ and transmissivity $\tau_{a b \nu}^{\prime \prime}\left[\mathbf{u}_{\mathbf{1}}, \mathbf{u}, \mathbf{n}_{a}\left(\mathbf{u}_{1}, \mathbf{r}_{0}\right)\right]$, generally given by the interfacial reflection-transmission laws, and on $f_{i \mathbf{u}_{1}}^{S(0)}\left(\mathbf{r}_{0}\right)$, i.e.

$$
\begin{aligned}
& p_{\nu a a}^{S(0)}\left(\mathbf{u}_{1}, \mathbf{u}\right) \frac{\mathrm{d} \Omega_{a}}{4 \pi}= \\
& \frac{\int_{\Pi_{a} V / \mathbf{u}_{1} \cdot \mathbf{n}_{a} \leq 0} f_{i \mathbf{u}_{1}}^{S(0)}\left(\mathbf{r}_{0}\right) \rho_{\nu a a}^{\prime \prime}\left[\mathbf{u}_{\mathbf{1}}, \mathbf{u}, \mathbf{n}_{a}\left(\mathbf{u}_{1}, \mathbf{r}_{0}\right)\right]\left[-\mathbf{u}_{\mathbf{1}} \cdot \mathbf{n}_{a}\left(\mathbf{u}_{1}, \mathbf{r}_{0}\right)\right] \mathrm{d} \mathbf{r}_{0} \mathrm{~d} \Omega_{a}}{\int_{0}^{4 \pi} \int_{\Pi_{a} V / \mathbf{u}_{1} \cdot \mathbf{n}_{a} \leq 0} f_{i \mathbf{u}_{1}}^{S(0)}\left(\mathbf{r}_{0}\right) \rho_{\nu a a}^{\prime \prime}\left[\mathbf{u}_{1}, \mathbf{u}^{\prime}, \mathbf{n}_{a}\left(\mathbf{u}_{1}, \mathbf{r}_{0}\right)\right]\left[-\mathbf{u}_{\mathbf{1}} \cdot \mathbf{n}_{a}\left(\mathbf{u}_{1}, \mathbf{r}_{0}\right)\right] \mathrm{d} \mathbf{r}_{0} \mathrm{~d} \Omega_{a}^{\prime}}(9)
\end{aligned}
$$

where $\mathbf{n}_{a}$ is the normal unit vector at the current impact point $N\left(\mathbf{u}_{1}, \mathbf{r}_{0}\right)$ oriented towards the phase a,

$$
\begin{aligned}
& p_{\nu a b}^{S(0)}\left(\mathbf{u}_{1}, \mathbf{u}\right) \frac{\mathrm{d} \Omega_{b}}{4 \pi}= \\
& \frac{\int_{\Pi_{a} V / \mathbf{u}_{1} \cdot \mathbf{n}_{a} \leq 0} f_{i \mathbf{u}_{1}}^{S(0)}\left(\mathbf{r}_{0}\right) \tau_{\nu a b}^{\prime \prime}\left[\mathbf{u}_{\mathbf{1}}, \mathbf{u}, \mathbf{n}_{a}\left(\mathbf{r}_{0}, \mathbf{u}_{1}\right)\right]\left[-\mathbf{u}_{\mathbf{1}} \cdot \mathbf{n}_{a}\left(\mathbf{r}_{0}, \mathbf{u}_{1}\right)\right] \mathrm{d} \mathbf{r}_{0} \mathrm{~d} \Omega_{b}}{\int_{0}^{4 \pi} \int_{\Pi_{a} V / \mathbf{u}_{1} \cdot \mathbf{n}_{a} \leq 0} f_{i}^{S(0)}\left(\mathbf{r}_{0}\right) \tau_{\nu a b}^{\prime \prime}\left[\mathbf{u}_{1}, \mathbf{u}^{\prime}, \mathbf{n}_{a}\left(\mathbf{r}_{0}, \mathbf{u}_{1}\right)\right]\left[-\mathbf{u}_{\mathbf{1}} \cdot \mathbf{n}_{a}\left(\mathbf{r}_{0}, \mathbf{u}_{1}\right)\right] \mathrm{d} \mathbf{r}_{0} \mathrm{~d} \Omega_{b}^{\prime}}(10)
\end{aligned}
$$


The phase function given by Eq.10 is expressed in the medium $\mathrm{b}$ of refractive index $n_{\nu b}$. According with the notations of Fig.1, the phase functions $p_{\nu a b b}^{S(2)}\left(\mathbf{u}_{1}, \mathbf{u}\right)$ and $p_{\nu a b a}^{S(2)}\left(\mathbf{u}_{1}, \mathbf{u}\right)$, the first ones corresponding to the transparent medium $\mathrm{b}$, are given by equations similar to Eqs.9-10, with the value of $\kappa_{\nu b}$ being set to 0 in Eq.8.

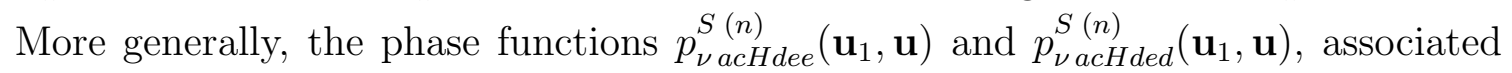
with the $n^{\text {th }}$ set of interfacial scattering events, i.e with the scattering cumulative probabilities $P_{s c \nu a c H d e e}^{S(n)}$ and $P_{s c \nu \text { acHded }}^{S(n)}$, depend both on the reflection-transmission laws on $f_{i \mathbf{u}_{1} \text { acHde }}^{S(n-1)}\left(\mathbf{r}_{n-1}\right)$, the spatial distribution function of the initial source points associated with the incidence directions $\mathbf{u}_{1}$. They write

$$
\begin{aligned}
& p_{\nu \text { acHdee }}^{S(n)}\left(\mathbf{u}_{\mathbf{1}}, \mathbf{u}\right) \frac{\mathrm{d} \Omega_{e}}{4 \pi}= \\
& \frac{\int_{\Sigma^{(n-1)} / \mathbf{u}_{1} \cdot \mathbf{n}_{e} \leq 0} f_{i \mathbf{u}_{1} a c H d e}^{S(n-1)}\left(\mathbf{r}_{n-1}\right) \rho_{\nu e e}^{\prime \prime}\left[\mathbf{u}_{\mathbf{1}}, \mathbf{u}, \mathbf{n}_{e}\left(\mathbf{u}_{1}, \mathbf{r}_{n-1}\right)\right]\left[-\mathbf{u}_{\mathbf{1}} \cdot \mathbf{n}_{e}\left(\mathbf{u}_{1}, \mathbf{r}_{n-1}\right)\right] \mathrm{d} \mathbf{r}_{n-1} \quad \mathrm{~d} \Omega_{e}}{\int_{0}^{4 \pi} \int_{\Sigma^{(n-1)} / \mathbf{u}_{1} \cdot \mathbf{n}_{e} \leq 0} f_{i \mathbf{u}_{1} a c H d e}^{S(n-1)}\left(\mathbf{r}_{n-1}\right) \rho_{\nu e e}^{\prime \prime}\left[\mathbf{u}_{\mathbf{1}}, \mathbf{u}^{\prime}, \mathbf{n}_{e}\left(\mathbf{u}_{1}, \mathbf{r}_{n-1}\right)\right]\left[-\mathbf{u}_{\mathbf{1}} \cdot \mathbf{n}_{e}\left(\mathbf{u}_{1}, \mathbf{r}_{n-1}\right)\right] \mathrm{d} \mathbf{r}_{n-1} \mathrm{~d} \Omega_{e}^{\prime}} \\
& p_{\nu a c H d e d}^{S(n)}\left(\mathbf{u}_{\mathbf{1}}, \mathbf{u}\right) \frac{\mathrm{d} \Omega_{d}}{4 \pi}= \\
& \frac{\int_{\Sigma^{(n-1)} / \mathbf{u}_{1} \cdot \mathbf{n}_{e} \leq 0} f_{i \mathbf{u}_{1} \text { acHde }}^{S(n-1)}\left(\mathbf{r}_{n-1}\right) \tau_{\nu e d}^{\prime \prime}\left[\mathbf{u}_{\mathbf{1}}, \mathbf{u}, \mathbf{n}_{e}\left(\mathbf{u}_{1}, \mathbf{r}_{n-1}\right)\right]\left[-\mathbf{u}_{\mathbf{1}} \cdot \mathbf{n}_{e}\left(\mathbf{r}_{n-1}, \mathbf{u}_{1}\right)\right] \mathrm{d} \mathbf{r}_{n-1} \mathrm{~d} \Omega_{d}}{\int_{0}^{4 \pi} \int_{\Sigma^{(n-1)} / \mathbf{u}_{1} \cdot \mathbf{n}_{e} \leq 0} f_{i \mathbf{u}_{1} \text { acHed }}^{S(n-1)}\left(\mathbf{r}_{n-1}\right) \tau_{\nu e d}^{\prime \prime}\left[\mathbf{u}_{\mathbf{1}}, \mathbf{u}^{\prime}, \mathbf{n}_{e}\left(\mathbf{u}_{1}, \mathbf{r}_{n-1}\right)\right]\left[-\mathbf{u}_{\mathbf{1}} \cdot \mathbf{n}_{e}\left(\mathbf{r}_{n-1}, \mathbf{u}_{1}\right)\right] \mathrm{d} \mathbf{r}_{n-1} \mathrm{~d} \Omega_{d}^{\prime}}
\end{aligned}
$$

The principle of determination of $f_{i \mathbf{u}_{1} \text { acHde }}^{S(n-1)}\left(\mathbf{r}_{n-1}\right)$, the spatial distribution function of the initial source points associated with the incidence direction $\mathbf{u}_{1}$ is exposed in Sec.2.1.4.

\subsubsection{Principles of implementation}

The radiative statistical functions of extinction, absorption and scattering and the phase functions are determined by a statistical Monte Carlo method at the limit of large numbers of generated power bundles. This approach is similar to the ones in use in Refs.[5, 8, 9, 14, 15, 17, 29]. The originality of the present work is due to the fact that a homogenised phase is non Beerian and characterised by non diffuse interfaces, more precisely interfaces which obey general interfacial laws for reflection and transmission. As developed in this Section, extinction, scattering and absorp- 
tion phenomena can then not be studied by using an intensity field, which implicitly globalises successive scattering events. Extinction, scattering and absorption have to be separately studied for every type of source term, associated with initial volume emission and successive interfacial scattering events.

The different phenomena associated with emission source terms are classically modeled as in the previous cited references: As the medium is assumed statistically homogeneous, all the source points are considered as isotropic and equivalent.

The successive sets of scattering events (n) are determined by following emission and scattering of generated power bundles. In these conditions, emission and scattering source terms are correlated to transmission and extinction by absorption or scattering. But it is classically assumed[16] that extinction by scattering is not correlated with the associated scattering source terms: Scattering source terms corresponding to the $n^{\text {th }}$ set of scattering events are represented by independently generated bundles, by following the scattering law characterised by a specific phase function, defined in Sec.2.1.3.

A huge number of emitted power bundles are first shot in random directions $\mathbf{u}_{1}$ from random volume points $M_{0}\left(\mathbf{r}_{0}\right)$ of a shooting zone of the real absorbing phase a. For the sake of clarity, only the first successive scattering events will be followed for illustrating the method: These chosen events are represented by bold lines in Fig.1. The nature of extinction of the emitted power bundles is determined by sampling of random numbers which allow Eq.4 to be verified: A part of the emitted power bundles characterised by $P_{a \nu a}^{S(0)}$ is absorbed within the phase, a second part characterised by $P_{s c \nu a a}^{S(0)}$ is internally scattered, the third one characterised by $P_{s c \nu a b}^{S(0)}$ is externally scattered. The part $\Sigma^{*(0)}$ of the interface area which is impacted by the bundles issued in the initial direction $\mathbf{u}$ from the set of points $M_{0}\left(\mathbf{r}_{0}\right)$ has been determined: The corresponding current points are $M_{0}^{*}\left(\mathbf{r}_{0}^{*}\right)$. The set of points $M_{0}^{*}\left(\mathbf{r}_{0}^{*}\right)$ of $\Sigma^{*(0)}$ are now source points for both internal and external scattering. The associated phase functions $p_{\nu a a}^{S(0)}$ and $p_{\nu a b}^{S(0)}$ are determined from Eqs.9 and 10.

By commodity, $M_{0}^{*}\left(\mathbf{r}_{0}^{*}\right)$ and $\Sigma^{*(0)}$ are now called $M_{n}\left(\mathbf{r}_{n}\right)$ and $\Sigma^{(n)}$ considered as sources of the $\mathrm{n}^{\text {th }}$ set of scattering events. In the case of internal scattering, with the notations of Fig.1, $M_{0}^{*}\left(\mathbf{r}_{0}^{*}\right)$ and $\Sigma^{*(0)}$ now become $M_{1}\left(\mathbf{r}_{1}\right)$ and $\Sigma^{(1)}$ and in the case of 
external scattering, $M_{2}\left(\mathbf{r}_{2}\right)$ and $\Sigma^{(2)}$. These last source points are characterised by a spatial scattering distribution function $f_{\mathbf{u} a b}^{S(2)}\left(\mathbf{r}_{2}\right)$ in the direction $\mathbf{u}$, which depends on $W_{\mathbf{u} a b}^{(2)}\left(\mathbf{r}_{2}\right)$, the interfacial density over $\Sigma^{(2)}$ of the bundles which are externally scattered in an elementary solid angle $\mathrm{d} \Omega$ around $\mathbf{u}$, i.e.

$$
f_{\mathbf{u} a b}^{S(2)}\left(\mathbf{r}_{2}\right)=\frac{W_{\mathbf{u} a b}^{(2)}\left(\mathbf{r}_{2}\right)}{\int_{\Sigma_{\mathbf{u}}^{(1)} / V / \mathbf{u} \cdot \mathbf{n}_{b}\left(\mathbf{r}_{2}^{\prime}\right) \geq 0} W_{\mathbf{u} a b}^{(2)}\left(\mathbf{r}_{2}^{\prime}\right) \mathrm{d} \mathbf{r}_{2}^{\prime}},
$$

where $\mathbf{n}_{b}$ is the normal unit vector at a current point $M_{2}\left(\mathbf{r}_{2}\right)$ of $\Sigma^{(2)}$, oriented towards the phase $b$.

The extinction cumulative distribution function $G_{e x t \nu a b}^{S(2)}$ within the phase b associated with the considered external scattering source term is then determined according to Eq.3 by using $f_{\mathbf{u} a b}^{S(2)}\left(\mathbf{r}_{2}\right)$ as weight function and an equation similar to Eq.1. By using a new set of random number samplings which allow Eqs.5 and 6 to be verified, the extinction of the externally scattered power bundles is defined as previously: A part of these bundles characterised by $P_{s c a b b}^{t S(2)}$ is internally scattered within the phase b, the second part characterised by $P_{s c a b a}^{t S(2)}$ is externally scattered towards the phase a. $\Sigma^{*(2)}$ is the part of the interfaces covered by the corresponding extinction points in an elementary solid angle $d \Omega_{b 1}$ around $\mathbf{u}_{1}$ : It is characterised by the interfacial density $W_{i \mathbf{u}_{1}}^{(2)}\left(\mathbf{r}_{2}^{*}\right)$ which is obtained by the Monte Carlo method. It allows $f_{i \mathbf{u}_{1} a b}^{S(2)}\left(\mathbf{r}_{2}^{*}\right)$, the spatial distribution function over $\Sigma^{*(2)}$ of the impact points associated with the incidence direction $\mathbf{u}_{1}$ to be determined by

$$
f_{i \mathbf{u}_{1} a b}^{S(2)}\left(\mathbf{r}_{2}^{*}\right)=\frac{W_{i \mathbf{u}_{1} a b}^{(2)}\left(\mathbf{r}_{2}^{*}\right)}{\int_{\Sigma_{\mathbf{u}_{1}}^{*(2)} / V / \mathbf{u}_{1} \cdot \mathbf{n}_{b}\left(\mathbf{r}_{2}^{\prime}{ }^{*}\right) \leq 0} W_{i \mathbf{u}_{1} a b}^{(2)}\left(\mathbf{r}_{2}^{\prime *}\right) d \mathbf{r}_{2}^{\prime}{ }^{*}},
$$

where $\mathbf{n}_{b}$ is the normal unit vector at a point $M_{2}^{*}\left(\mathbf{r}_{2}^{*}\right)$ of $\Sigma^{*(2)}$, oriented towards the phase b. $f_{i \mathbf{u}_{1} a b}^{S(2)}\left(\mathbf{r}_{2}^{*}\right)$ allows the phase functions $p_{\nu a b b}^{S(2)}$ and $p_{\nu a b a}^{S(2)}$ to be determined from Eqs.11,12.

The previous procedure is iterated for the successive sets of scattering events. As previously, $M_{2}^{*}\left(\mathbf{r}_{2}^{*}\right)$ and $\Sigma^{*(2)}$ become $M_{3}\left(\mathbf{r}_{3}\right)$ and $\Sigma^{(3)}$ sources of the internal scattering events of the type abb and $M_{4}\left(\mathbf{r}_{4}\right)$ and $\Sigma^{(4)}$ sources of the external scattering 
events of the type aba, etc.

\subsection{Coupled Generalised Radiative Transfer Equations}

Radiative transfer through non Beerian homogenised phases is modeled by coupled integral Generalised Radiative Transfer Equations (GRTEs), which account for the memory effect characterising these media. The only initial source is, in the present case, the volume emission by a. The first scattering source terms are associated with this initial volume emission: $S_{s c \nu a a}^{S(1)}$ corresponds to internal scattering within a and $S_{s c \nu a b}^{S(2)}$ to external scattering from a to b and write

$$
\begin{array}{ll}
c=a, b & S_{s c \nu a c}^{S(i)}\left(\mathbf{u}, s^{\prime}\right)= \\
i=1,2 & \int_{4 \pi} \int_{s_{1 w}}^{s_{1}^{\prime}} \frac{\mathrm{d} P_{s c \nu a c}^{S(i)}}{\mathrm{d} s_{1}^{\prime}}\left(\mathbf{u}_{1}, s_{1}^{\prime}-s_{1}\right) \frac{p_{\nu a c}^{S(i)}\left(\mathbf{u}_{1}, \mathbf{u}\right)}{4 \pi} \prod_{a} \kappa_{\nu a} n_{\nu a}^{2} I_{\nu a}^{\circ}\left[T_{a}\left(s_{1}\right)\right] \mathrm{d} s_{1} \mathrm{~d} \Omega_{1 a} .
\end{array}
$$

A current scattering source term, associated with a set acHde is expressed from the recurrent equation

$S_{s c \nu a c H d e}^{S(n)}\left(\mathbf{u}, s^{\prime}\right)=\int_{4 \pi} \int_{s_{1 w}}^{s_{1}^{\prime}} \frac{\mathrm{d} P_{s c \nu a c H d e}^{S(n)}}{\mathrm{d} s_{1}^{\prime}}\left(\mathbf{u}_{1}, s_{1}^{\prime}-s_{1}\right) \frac{p_{\nu a c H d e}^{S(n)}\left(\mathbf{u}_{\mathbf{1}}, \mathbf{u},\right)}{4 \pi} S_{s c \nu c H d}^{S(n-1)}\left(\mathbf{u}_{1}, s_{1}\right) \mathrm{d} s_{1} \mathrm{~d} \Omega_{1 e}$,

where $S_{s c \nu a c H d}^{S(n-1)}$ is the source term preceding $S_{s c \nu a c H d e}^{S(n)}$ in the set acHde.

The Generalised Radiative Transfer Equation (GRTE) of the phase a writes, in integral formulation, by generalising the expression of Ref.[3]

$$
\begin{aligned}
I_{\nu a}\left(\mathbf{u}, s^{\prime}\right)= & I_{\nu a}\left(\mathbf{u}, s_{w}\right)\left[1-G_{\text {ext } \nu a}^{S_{w}}\left(\mathbf{u}, s^{\prime}-s_{w}\right)\right] \\
+ & \int_{s_{w}}^{s^{\prime}}\left(\kappa_{\nu a} \Pi_{a} n_{\nu a}^{2} I_{\nu}^{\circ}\left[T_{a}(s)\right]\left[1-G_{\text {ext } \nu a}\left(\mathbf{u}, s^{\prime}-s\right)\right]\right. \\
& \left.+\sum_{a c H d a} S_{s c \nu a c H d a}^{S}(\mathbf{u}, s)\left[1-G_{\text {ext } \nu a c H d a}^{S}\left(\mathbf{u}, s^{\prime}-s\right)\right]\right) \mathrm{d} s
\end{aligned}
$$

where $I_{\nu a}$ is the intensity within the medium a of refractive index $n_{\nu a}$ defined per unit volume of the whole porous medium: $I_{\nu a}$ is then proportional to the volume

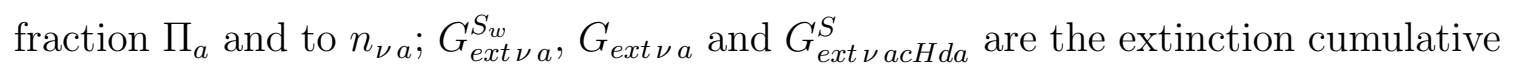
distribution functions associated with the different types of source terms: Intensity 
distribution $I_{\nu a}\left(\mathbf{u}, s_{w}\right)$ at the porous medium boundary point of abscissa $s_{w}$, volume isotropic emission by a, set of interfacial scattering source terms within or towards a, including $S_{s c \nu a a}^{S(1)}(\mathbf{u}, s)$.

The GRTE of the phase b is similar to Eq.17 without emission term

$$
\begin{gathered}
I_{\nu b}\left(\mathbf{u}, s^{\prime}\right)=I_{\nu b}\left(\mathbf{u}, s_{w}\right)\left[1-P_{s c \nu b}^{t S_{w}}\left(\mathbf{u}, s^{\prime}-s_{w}\right)\right] \\
+\int_{s_{w}}^{s^{\prime}}\left(\sum_{a c H d b} S_{s c \nu a c H d b}^{S}(\mathbf{u}, s)\left[1-P_{s c \nu a c H d b}^{t S}\left(\mathbf{u}, s^{\prime}-s\right)\right]\right) \mathrm{d} s
\end{gathered}
$$

As b is a transparent medium, any extinction cumulative distribution function $G_{e x t}^{S}$ of the previous equation simply is a scattering cumulative probability $P_{s c}^{t S}$.

When $\mathrm{b}$ is a Beerian homogenised phase, Equation 18 simply becomes

$$
\begin{gathered}
I_{\nu b}\left(\mathbf{u}, s^{\prime}\right)=I_{\nu b}\left(\mathbf{u}, s_{w}\right) \exp \left(-\left[\sigma_{\nu b a}(\mathbf{u})+\sigma_{\nu b b}(\mathbf{u})\right]\left(s^{\prime}-s_{w}\right)\right) \\
+\int_{s_{w}}^{s^{\prime}}\left[\int_{0}^{4 \pi} \sigma_{\nu b b}\left(\mathbf{u}_{1}\right) \frac{p_{\nu b b}\left(\mathbf{u}_{1}, \mathbf{u}\right)}{4 \pi} I_{\nu b}\left(\mathbf{u}_{1}, s\right) \mathrm{d} \Omega_{b 1}+\int_{0}^{4 \pi} \sigma_{\nu a b}\left(\mathbf{u}_{1}\right) \frac{p_{\nu a b}\left(\mathbf{u}_{1}, \mathbf{u}\right)}{4 \pi} I_{\nu a}\left(\mathbf{u}_{1}, s\right) \mathrm{d} \Omega_{a 1}\right] \\
\mathrm{x} \exp \left(-\left[\sigma_{\nu b a}(\mathbf{u})+\sigma_{\nu b b}(\mathbf{u})\right]\left(s^{\prime}-s\right)\right) \mathrm{d} s
\end{gathered}
$$

where $\sigma_{\nu c d}(\mathbf{u})$ and $p_{\nu c d}(\mathbf{u})$ are the scattering coefficient and phase function associated with global scattering from the phase $\mathrm{c}$ ( $\mathrm{a}$ or b) to the phase $\mathrm{d}$ ( $\mathrm{a}$ or b). Indeed, for this Beerian homogenised phase the contributions of all the successive scattering events have merged in the local intensities $I_{\nu a}$ and $I_{\nu b}$. Note also that in Eq.19, the emission source term is implicitly accounted for in the expression of $I_{\nu a}$.

\subsection{Coupled RTEs}

When a homogenised phase is non Beerian, it is only characterised in the general case by a Generalised Radiative Transfer Equation. A classical Radiative Transfer Equation (RTE) nevertheless has a physical meaning when the validity conditions of a radiative Fourier's law are fulfilled[14, 16].

Introducing the smallest distance $\delta$ along which the medium is optically thick, two sufficient validity conditions have been defined in Ref.[30] for a unique propagation phase: i) The Fourier's model is only valid in zones at a distance larger than $\delta$ 
from the boundaries of the whole porous medium; ii) The temperature $T$ must be practically uniform over $\delta$. An accurate quantitative criterion has been given in the previous reference

$$
\left|\frac{1}{T} \frac{\partial T}{\partial x}\right|<\eta \kappa^{e f f}(\omega, g),
$$

where $\kappa^{e f f}$ is an effective absorption coefficient accounting for multiple scattering events ( $\omega$ and $g$ are the albedo and the anisotropy factor, respectively) and $\eta$ a coefficient depending on $g$ and $\omega$. As $\kappa^{e f f}$ is proportional to the absorption coefficient of the phase, the condition is not fulfilled for a transparent phase. In the present case of a medium with transparent and semi transparent phases, a model based on two coupled RTEs will be developed and its validity discussed by comparison with the results of the two coupled reference GRTEs. In these conditions, the homogenised phases become Beerian, as physically discussed in Ref.[3]. The temperature is only defined for the absorbing phase, from the radiation point of view.

The previous assumptions are assumed valid for the two phases. Consequently:

- Radiation transfer is locally ruled by the intensities $I_{\nu a}$ and $I_{\nu b}$. The modeling is then simplified as the intensities gather all the types of incident scattered radiations at a given point and in a given direction;

- A summation over $\delta$ of the radiative statistical functions is considered as a summation over an infinite distance.

- All the emission and scattering source terms are assumed uniform along the distance $\delta$ within a or b respectively.

- The intensities $I_{\nu a}(\mathbf{u})$ and $I_{\nu b}(\mathbf{u})$ within a and b also are uniform along the distance $\delta$ and linked to the global source terms $S_{\nu a}^{B}(\mathbf{u})$ and $S_{\nu b}^{B}(\mathbf{u})$ given by

$$
\begin{aligned}
& S_{\nu a}^{B}(\mathbf{u})=\Pi_{a} \kappa_{\nu a} n_{\nu a}^{2} I_{\nu}^{\circ}\left(T_{a}\right)+S_{s c \nu a a}^{B}(\mathbf{u})+S_{s c \nu b a}^{B}(\mathbf{u}) ; \\
& S_{\nu b}^{B}(\mathbf{u})=S_{s c \nu a b}^{B}(\mathbf{u})+S_{s c \nu b b}^{B}(\mathbf{u}) .
\end{aligned}
$$

The source terms involved in the previous equations gather all the contributions of the successive scattering events of given types.

Under these assumptions, the RTEs for the phases a and b can be expressed vs the intensities $I_{\nu a}$ and $I_{\nu b}$. As the homogenised phases are assumed Beerian, there 
is no difference between the statistical quantities associated with the volume sources and all the interfacial sources of the same types[3]. As discussed in this last reference, the best accuracy is obtained by determining $G_{e x t \nu c}$ associated with isotropic volume source points. Far from the boundaries of the porous medium, Equations 17,18 are in these conditions replaced by simple RTEs, i.e.

$$
\begin{aligned}
c=a, b \quad I_{\nu c}(\mathbf{u}) & =S_{\nu c}^{B}(\mathbf{u}) \int_{s-\delta}^{s}\left[1-G_{\text {ext } \nu c}(\mathbf{u}, v)\right] \mathrm{d} v \\
& =S_{\nu c}^{B}(\mathbf{u}) \int_{0}^{\infty}\left[1-G_{e x t \nu c}(\mathbf{u}, v)\right] \mathrm{d} v=S_{\nu c}^{B}(\mathbf{u}) / B_{\nu c}(\mathbf{u})
\end{aligned}
$$

where $B_{\nu a}(\mathbf{u})$ and $B_{\nu b}(\mathbf{u})$ are generalised extinction coefficients at equilibrium defined by

$$
B_{\nu a}(\mathbf{u})=\left(\int_{0}^{\infty}\left[1-G_{e x t \nu a}(\mathbf{u}, v)\right] \mathrm{d} v\right)^{-1}, \quad B_{\nu b}(\mathbf{u})=\left(\int_{0}^{\infty}\left[1-P_{s c \nu b}(\mathbf{u}, v)\right]\right)^{-1} .
$$

The variations of the intensities and source terms are now accounted for at a scale $s^{\prime}$ larger than $\delta$ : The source terms do not any more compensate the extinction terms $-B_{\nu c}\left(\mathbf{u}, s^{\prime}\right) I_{\nu c}(\mathbf{u})$.

Under the assumptions of this Section, the internal and external scattering source terms $S_{s c \nu a a}\left(\mathbf{u}, s^{\prime}\right), S_{s c \nu a b}\left(\mathbf{u}, s^{\prime}\right), S_{s c \nu b a}\left(\mathbf{u}, s^{\prime}\right)$ and $S_{s c \nu b b}\left(\mathbf{u}, s^{\prime}\right)$ are given, for c=a,b and $\mathrm{d}=\mathrm{a}, \mathrm{b}$, by

$S_{s c \nu c d}\left(\mathbf{u}, s^{\prime}\right)=\int_{0}^{4 \pi} \int_{-\infty}^{s_{1}^{\prime}} B_{\nu c}\left(\mathbf{u}_{1}\right) I_{\nu c}\left(\mathbf{u}_{1}, s_{1}\right) \frac{\mathrm{d} P_{s c \nu c d}}{\mathrm{~d} s^{\prime}}\left(\mathbf{u}_{1}, s_{1}^{\prime}-s_{1}\right) \frac{p_{\nu c d}\left(\mathbf{u}_{1}, \mathbf{u}\right)}{4 \pi} \mathrm{d} s_{1} \mathrm{~d} \Omega_{1 c}$,

in which the phase functions $p_{\nu c d}$ are independent of the incidence direction distribution [3]. Note that, in Eq.24, $s^{\prime}$ and $s_{1}^{\prime}$ respectively are the abscissas of the same point $M^{\prime}$ in the direction $\mathbf{u}$ and in the current direction $\mathbf{u}_{1}$. Equation 24 then becomes

$$
S_{s c \nu c d}\left(\mathbf{u}, s^{\prime}\right)=\int_{0}^{4 \pi} \Sigma_{\nu c d}\left(\mathbf{u}_{1}\right) I_{\nu c}\left(\mathbf{u}_{1}, s_{1}^{\prime}\right) \frac{p_{\nu c d}\left(\mathbf{u}_{1}, \mathbf{u}\right)}{4 \pi} \mathrm{d} \Omega_{1 c}
$$


where $\Sigma_{\nu c d}\left(\mathbf{u}_{1}\right)$, equal to $P_{s c \nu c d}\left(\mathbf{u}_{1}, \infty\right) B_{\nu c}\left(\mathbf{u}_{1}\right)$, is the Beerian scattering coefficient associated with scattering from $\mathrm{c}$ towards $\mathrm{d}$.

The spatial and directional variations of the intensities at these large scales are then ruled by the RTEs of phases a and b, which write

$$
\begin{gathered}
\frac{\mathrm{d} I_{\nu a}}{\mathrm{~d} s^{\prime}}\left(\mathbf{u}, s^{\prime}\right)+B_{\nu a}(\mathbf{u}) I_{\nu a}\left(\mathbf{u}, s^{\prime}\right)=\prod_{a} \kappa_{\nu a} n_{\nu a}^{2} I_{\nu a}^{\circ}\left[T_{a}\left(s^{\prime}\right)\right]+S_{s c \nu a a}\left(\mathbf{u}, s^{\prime}\right)+S_{s c \nu b a}\left(\mathbf{u}, s^{\prime}\right) . \\
\frac{\mathrm{d} I_{\nu b}}{\mathrm{~d} s^{\prime}}\left(\mathbf{u}, s^{\prime}\right)+B_{\nu b}(\mathbf{u}) I_{\nu b}\left(\mathbf{u}, s^{\prime}\right)=S_{s c \nu a b}\left(\mathbf{u}, s^{\prime}\right)+S_{s c \nu b b}\left(\mathbf{u}, s^{\prime}\right) .
\end{gathered}
$$

Due to the equilibrium properties of the medium, it is shown in Appendix A (Eqs.A.9 and A.11) that

$$
\Sigma_{\nu d c}(-\mathbf{u})=\Sigma_{\nu d c}(\mathbf{u}) .
$$

and that Equation 25, the result of which is expressed in medium d, simply becomes (see Eqs. A.6, A.10)

$$
S_{s c \nu c d}\left(\mathbf{u}, s^{\prime}\right)=\left(\frac{n_{\nu d}^{2} \Pi_{d}}{n_{\nu c}^{2} \Pi_{c}}\right) \Sigma_{\nu d c}(\mathbf{u}) \int_{0}^{4 \pi} I_{\nu c}\left(\mathbf{u}_{1}, s_{1}^{\prime}\right) \frac{p_{\nu d c}\left(-\mathbf{u},-\mathbf{u}_{1}\right)}{4 \pi} \mathrm{d} \Omega_{1 c}\left(-\mathbf{u}_{1}\right) .
$$

Another consequence is that, in ITE conditions, the radiative power globally scattered from a toward $\mathrm{b}$ in direction $\mathbf{u}$ is equal to the one scattered from $\mathrm{b}$ toward $\mathrm{a}$ in direction $-\mathbf{u}$, i.e.

$$
S_{s c \nu c d}^{\circ}\left(\mathbf{u}, s^{\prime}\right)=S_{s c \nu d c}^{\circ}\left(-\mathbf{u}, s^{\prime}\right)
$$

which simply leads to

$$
n_{\nu a}^{2} \Pi_{a} \Sigma_{\nu a}(\mathbf{u})=n_{\nu b}^{2} \Pi_{b} \Sigma_{\nu b}(-\mathbf{u}) .
$$

The previous equality is valid for external scattering and internal scattering (case 
$\mathrm{c}=\mathrm{d})$. Finally, the two coupled RTE's related to a and b also write

$$
\begin{aligned}
\frac{\mathrm{d} I_{\nu a}}{\mathrm{~d} s^{\prime}}\left(\mathbf{u}, s^{\prime}\right) & +B_{\nu a}(\mathbf{u}) I_{\nu a}\left(\mathbf{u}, s^{\prime}\right)=\kappa_{\nu a} \Pi_{a} n_{\nu a}^{2} I_{\nu}^{\circ}\left[T_{a}\left(s^{\prime}\right)\right] \\
& +\Sigma_{\nu a a}(\mathbf{u}) \int_{0}^{4 \pi} I_{\nu a}\left(\mathbf{u}_{1}, s_{1}^{\prime}\right) \frac{p_{\nu a a}\left(-\mathbf{u},-\mathbf{u}_{1}\right)}{4 \pi} \mathrm{d} \Omega_{1 a}\left(-\mathbf{u}_{1}\right) \\
& +\left(\frac{n_{\nu a}^{2} \Pi_{a}}{n_{\nu b}^{2} \Pi_{b}}\right) \Sigma_{\nu a b}(\mathbf{u}) \int_{0}^{4 \pi} I_{\nu b}\left(\mathbf{u}_{1}, s_{1}^{\prime}\right) \frac{p_{\nu a b}\left(-\mathbf{u},-\mathbf{u}_{1}\right)}{4 \pi} \mathrm{d} \Omega_{1 b}\left(-\mathbf{u}_{1}\right)(32) \\
\frac{\mathrm{d} I_{\nu b}}{\mathrm{~d} s^{\prime}}\left(\mathbf{u}, s^{\prime}\right)+ & B_{\nu b}(\mathbf{u}) I_{\nu b}\left(\mathbf{u}, s^{\prime}\right)=\Sigma_{\nu b b}(\mathbf{u}) \int_{0}^{4 \pi} I_{\nu b}\left(\mathbf{u}_{1}, s_{1}^{\prime}\right) \frac{p_{\nu b b}\left(-\mathbf{u},-\mathbf{u}_{1}\right)}{4 \pi} \mathrm{d} \Omega_{1 b}\left(-\mathbf{u}_{1}\right) \\
+ & \left(\frac{n_{\nu b}^{2} \Pi_{b}}{n_{\nu a}^{2} \Pi_{a}}\right) \Sigma_{\nu b a}(\mathbf{u}) \int_{0}^{4 \pi} I_{\nu a}\left(\mathbf{u}_{1}, s_{1}^{\prime}\right) \frac{p_{\nu b a}\left(-\mathbf{u},-\mathbf{u}_{1}\right)}{4 \pi} \mathrm{d} \Omega_{1 a}\left(-\mathbf{u}_{1}\right) .
\end{aligned}
$$

In ITE conditions, these equations simply degenerate, by using the normalisation equation of the phase functions, into

$$
B_{\nu a}(\mathbf{u})=\kappa_{\nu a}+\Sigma_{\nu a a}(\mathbf{u})+\Sigma_{\nu a b}(\mathbf{u}) ; \quad B_{\nu b}(\mathbf{u})=\Sigma_{\nu b a}(\mathbf{u})+\Sigma_{\nu b b}(\mathbf{u}),
$$

equations which express the energy conservation between extinction and source terms.

\subsection{Radiative conductivity tensors}

The aim of this study is to determine $P_{a}^{R}$, the radiative power in the absorbing phase a per unit volume of the whole porous medium. Note that $P_{b}^{R}$, the corresponding quantity in phase $\mathrm{b}$, is zero as this phase is transparent. By using the previous coupled GRTEs or coupled RTEs, $P_{a}^{R}$ and $P_{b}^{R}$ are directly obtained from the knowledge of the intensity fields $I_{\nu a}$ and $I_{\nu b}$ within the two phases. But, in the present Section, the Fourier's model, when it is valid, is limited to the knowledge of the radiative fluxes within the phases a and $\mathrm{b}$, more precisely to the knowledge of the conductivity tensors of a and $\mathrm{b}$.

In terms of fluxes, the radiative balance of a volume element of a phase c, which leads to the determination of $P_{c}^{R}$, not only involves the fluxes through boundaries 
belonging to the considered phase, characterised by the Fourier's laws, but also the exchanged fluxes between the phase $\mathrm{c}$ and the other phase, through other boundaries. These exchanged fluxes are not zero as the intensities of the two phases in vacuum differ.

This study can only be achieved for a defined system with defined boundary conditions. It will be done in Section 4.2 for a felt of fibres. The present Section is limited to the determination of the radiative fluxes following a Fourier's law within the phases $a$ and $b$.

The two coupled RTEs are solved by a perturbation technique which generalises the method in use in Ref.[14], in which more details are given. The perturbation parameters are $\zeta_{a}=1 /\left[B_{\nu a}(\mathbf{u}) \delta\right]$ and $\zeta_{b}=1 /\left[B_{\nu b}(\mathbf{u}) \delta\right]$, where $\delta$ is the smallest spatial scale for which the two homogenised phases are optically thick. Non dimensional Equations 26 and 27 then write, by using $s^{\prime}=s^{\prime} / \delta$

$$
\begin{gathered}
\zeta_{a} \frac{\mathrm{d} I_{\nu a}}{\mathrm{~d} s^{\prime+}}\left(\mathbf{u}, s^{\prime}\right)+I_{\nu a}\left(\mathbf{u}, s^{\prime}\right)=\frac{\kappa_{\nu a}}{B_{\nu a}(\mathbf{u})} \Pi_{a} n_{\nu a}^{2} I_{\nu}^{\circ}\left[T_{a}\left(s^{\prime}\right)\right]+\frac{\left[S_{s c \nu a a}\left(\mathbf{u}, s^{\prime}\right)+S_{s c \nu b a}\left(\mathbf{u}, s^{\prime}\right)\right]}{B_{\nu a}(\mathbf{u})} \\
\zeta_{b} \frac{\mathrm{d} I_{\nu b}}{\mathrm{~d} s^{\prime}+}\left(\mathbf{u}, s^{\prime+}\right)+I_{\nu b}\left(\mathbf{u}, s^{\prime}\right)=\frac{\left[S_{s c \nu a b}\left(\mathbf{u}, s^{\prime}\right)+S_{s c \nu b b}\left(\mathbf{u}, s^{\prime}\right)\right]}{B_{\nu b}(\mathbf{u})}
\end{gathered}
$$

The intensities $I_{\nu a}$ and $I_{\nu b}$ write, in the perturbation method,

$$
c=a, b \quad I_{\nu c}=I_{\nu c}^{(0)}+I_{\nu c}^{(1)},
$$

where $I_{\nu c}^{(0)}$ and $I_{\nu c}^{(1)}$ are the solutions at the perturbation orders 0 and 1: $I_{\nu c}^{(0)}$ is the intensity in conditions of Local Thermal Equilibrium of radiation, $I_{\nu c}^{(1)}$ its perturbation due to the transport terms of Eqs.35 and 36 (see for instance Refs.[14, 31]).

In this particular case of a medium with a transparent phase and a semi transparent one, $I_{\nu c}^{(0)}$ is equal to the equilibrium intensity $\Pi_{c} n_{\nu c}^{2} I_{\nu}^{\circ}\left[T_{a}\left(s^{\prime}\right)\right]$, which, due to its isotropy, does not contribute to the radiative flux through the phase c. In these 
conditions, the flux through a phase c (a or b) writes

$$
c=a, b \quad \mathbf{q}_{c}^{R}\left(s^{\prime}\right)=\int_{0}^{\infty} \int_{0}^{2 \pi} \int_{0}^{\pi} I_{\nu c}^{(1)}\left(\mathbf{u}, s^{\prime}\right) \mathbf{u}(\theta, \phi) \mathrm{d} \theta \mathrm{d} \phi \mathrm{d} \nu .
$$

As shown in Ref.[14], the emission term in Eq.35 is of order 0. The transport terms involving temperature gradients in Eqs.35 and 36 are proportional to the perturbation parameters. In the determination of the solutions of order 1 of the coupled RTEs, $\mathrm{d} I_{\nu a} / \mathrm{d} s^{\prime}$ and $\mathrm{d} I_{\nu b} / \mathrm{d} s^{\prime}$ are taken at order 0 ; On the contrary, the extinction scattering source terms are taken at order $1 . I_{\nu a}^{(1)}$ and $I_{\nu b}^{(1)}$ are then iteratively obtained by solving the coupled integral equations

$$
c=a, b \quad I_{\nu c}^{(1) n+1}=\mathcal{L}_{c}\left(I_{\nu a}^{(1) n}, I_{\nu b}^{(1) n}\right)
$$

where $n$ is the iteration step and the quantities $\mathcal{L}_{c}(X, Y)$ are functionals defined, for $c=a, b$, by

$$
\mathcal{L}_{c}(X, Y)=-\frac{1}{B_{\nu c}(\mathbf{u})}\left(n_{\nu c}^{2} \frac{\partial I_{\nu}^{\circ}\left(T_{a}\right)}{\partial T_{a}} u_{i} \frac{\partial T_{a}}{\partial x_{i}^{\prime}}+S_{s c \nu c a}(X, Y)+S_{s c \nu c b}(X, Y)\right) .
$$

In Eq.40, the derivation vs $s^{\prime}$ has been replaced in cartesian tensorial notations by the operator $u_{i} \partial / \partial x_{i}^{\prime}$. It appears that, after convergence, $I_{\nu a}^{(1)}$ and $I_{\nu b}^{(1)}$ and consequently from Eq.38 the fluxes within a and $\mathrm{b}$ are proportional to $-u_{i} \partial T_{a} / \partial x_{i}^{\prime}$, i.e. are given by radiative Fourier's laws

$$
c=a, b \quad \mathbf{q}_{c i}^{R}=-k_{c i j}^{R} \frac{\partial T_{a}}{\partial x_{j}^{\prime}}
$$

valid if the precise conditions of Gomart and Taine [30] discussed in Sec.2.3 are fulfilled.

\section{Characterisation of a felt of fibres}

The material of interest is a felt of absorbing but non scattering fibres made of a refractory ceramic material such as alumina or zirconia. For this study, the calculations are performed over a representative computer-generated virtual fibre felt 
arrangement. More precisely, the phase a is made of overlapping fibres, cylinders of diameter $d$ and length $L$, equal to $100 d$, and the transparent phase $\mathrm{b}$ of refractive index $n_{b}$ occupies the void space. The centers of the cylinders have random locations, the unit vectors of their axes $\mathbf{u}_{C}\left(\theta_{C}, \phi_{C}\right)$ are characterised by random values of $\phi_{C}$ and a Gaussian distribution of $\theta_{C}$ centered in the plane normal to the $z$ axis (see Fig.2). Total specular reflection conditions are imposed at boundaries parallel to the $z$ axis (see Fig.2): The medium is then infinite along the $x$ and $y$ axes.

The fibres of refractive index $n_{a}$ and of absorption coefficient $\kappa_{a}$ have smooth surfaces and are assumed gray. Due to the large value of their refractive index, the fibres are characterised by strongly anisotropic reflection and transmission laws and an important total reflection phenomenon occurs within these fibres. The simple model of diffuse interfacial laws cannot be applied in these conditions. The fibre bidirectional reflectivity and transmissivity are in the following defined by the Fresnel's laws for unpolarised radiation, only depending on $n_{a}$ and $n_{b}$ as $\chi_{a}$ the imaginary part of the complex optical index of the fibers is assumed small compared to $n_{a}$ and $n_{b}$. A typical value of $\kappa_{a} d$ for the considered fibres is 0.3 , which typically corresponds to $d=20 \mu \mathrm{m}$.

The real interfacial radiative properties depend on the fibre rugosity compared to the range of radiation wavelength: In many cases they present a diffuse part and a specular one. For the sake of simplicity, the Fresnel?s reflection and transmission laws corresponding to smooth interfaces have been considered in Sec. 3 . 


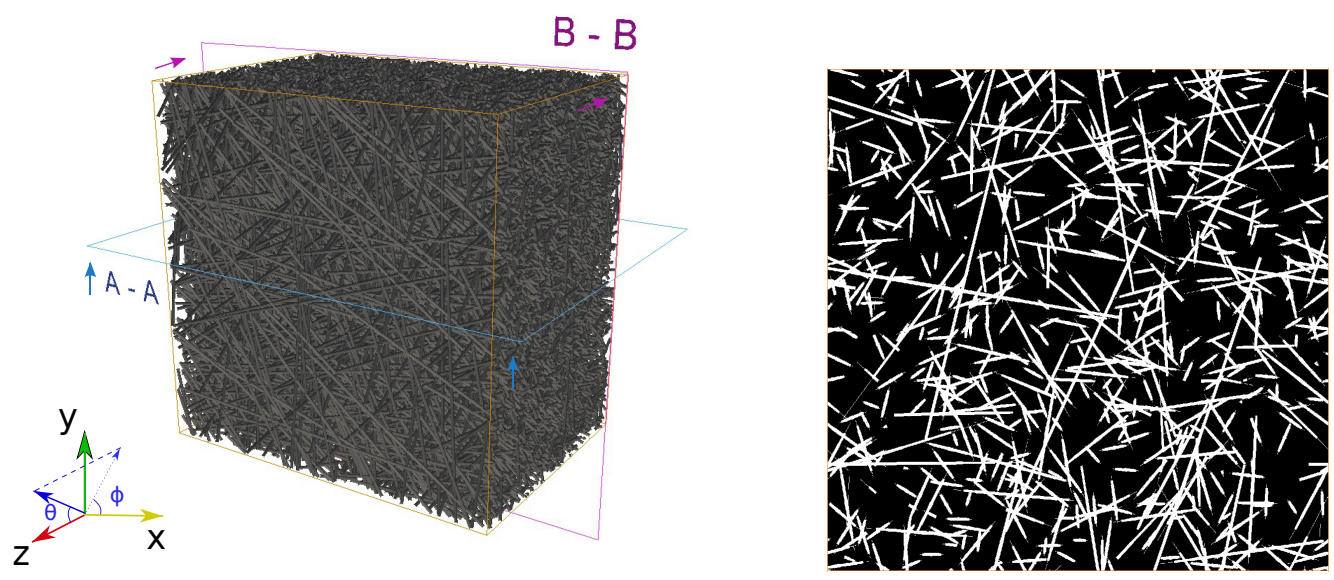

Figure 2: Left: Generated fibrous medium $\left(\Pi_{b}=0.75\right)$; Right: Cross section BB normal to the $z$ axis.

\subsection{Numerical implementation}

The radiative statistical functions defined in Sec.2.1, i.e. $G_{\text {exta }}\left(\mathbf{u}, s^{\prime}-s\right), P_{\text {scaa }}\left(\mathbf{u}, s^{\prime}-\right.$ $s), P_{s c a b}\left(\mathbf{u}, s^{\prime}-s\right), p_{a a}\left(\mathbf{u}_{1}, \mathbf{u}\right), p_{a b}\left(\mathbf{u}_{1}, \mathbf{u}\right)$, and $G_{\text {extc }}^{S(n)}\left(\mathbf{u}, s^{\prime}-s\right), P_{s c c d}^{S(n)}\left(\mathbf{u}, s^{\prime}-s\right)$ and $p_{c d}^{S(n)}\left(\mathbf{u}_{1}, \mathbf{u}\right)$, are directly determined by a Monte Carlo method from morphology data and the Fresnel's laws. A huge number of power bundles, which represent the emission within the volume of the cylindrical fibres, are shot and followed up to their absorption within a fibre (phase a), after possible multiple internal or external scattering events. Both absorption and scattering events are stochastically modeled. The calculations are carried out in the following manner:

i) Many numerical fibrous media have been generated. For any generated medium, a shooting zone representative of this medium, which is statistically homogeneous at large scale but strongly anisotropic at any scale, has been defined. A huge number of rays have then been shot from random volume points of the phase of such a zone. In the case presented in Fig.2, 40 numerical media have been generated, and $10^{10}$ rays have been shot from any associated shooting zone, cube of edge equal to $30 d$.

ii) For any medium a calculation zone ( extinction zone), including the shooting one, is chosen such that at its boundaries all $G_{e x t}$ functions are larger than 0.99. The calculation zone associated with the case of Fig.2 is a parallelepiped of length $\mathcal{L}=100 \mathrm{~d}$ along $z$ and of squared cross section of edge $\mathcal{D}=100 d$ along $x$ and $y$. 
A shot $t$ is characterised by an emission point $M_{t}$, of coordinates defined from three random numbers within a randomly chosen cylinder of the shooting zone. The ray direction $\mathbf{u}(\theta, \phi)$ is defined by two other random numbers associated with $\mu=\cos \theta$ and $\phi$. More precisely, the directional space is divided in 41 equal intervals along $\mu$ varying in $[-1,1]$ and 101 intervals along $\phi$ varying in $[0,2 \pi]$.

Due to the symmetry of the medium, all the results only depend on $\mu$. They are averaged over $\phi$, which accelerates the convergence: Indeed, radiative transfer only occurs along $z$. Moreover, due to the medium planar symmetry, the results will be presented in the variation range $[0,1]$ of $\mu$ for the extinction cumulative distribution functions and scattering cumulative probabilities and of $\mu_{1}$ and $\mu$ for the phase functions, as detailed in Sec.3.2.

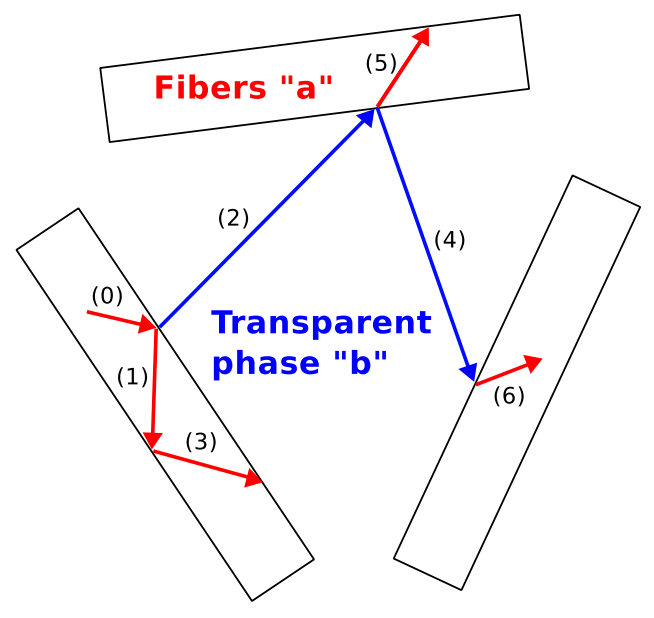

Figure 3: Example of radiative paths.

As detailed in Sec.2.1.4, $G_{\text {exta }}(\mathbf{u}), P_{\text {scaa }}(\mathbf{u}), P_{\text {scab }}(\mathbf{u}), p_{a a}\left(\mathbf{u}_{1}, \mathbf{u}\right)$ and $p_{a b}\left(\mathbf{u}_{1}, \mathbf{u}\right)$ are first determined by following the set, called 0 , of all rays emitted by fibres a in the direction $\mathbf{u}$ (see Fig.3). The calculation associated with a given ray is stopped in case of absorption. In case of external scattering (path 2 of Fig.3) $P_{\text {scab }}(\mathbf{u})$ and $p_{a b}^{S}\left(\mathbf{u}_{1}, \mathbf{u}\right)$ are incremented and calculations are now carried out in order to increment $G_{e x t a b}^{S(2)}$ and possibly $P_{s c a b a}^{S(2)}$ and $p_{s c a b a}^{S(2)}$, or $P_{s c a b b}^{S(2)}$ and $p_{s c a b b}^{S(2)}$, etc.

The relative standard deviations on the quantities associated with initial emis- 
sion by the phase a, i.e. $G_{\text {exta }}(\mathbf{u}), P_{a a}(\mathbf{u}), P_{s c a}(\mathbf{u}), P_{s c a a}^{S}(\mathbf{u}), P_{s c a b}^{S}(\mathbf{u}), p_{a a}^{S}\left(\mathbf{u}_{1}, \mathbf{u}\right)$, $p_{a b}^{S}\left(\mathbf{u}_{1}, \mathbf{u}\right)$ are lower than $1 \%$. They have been obtained from $10^{9}$ realisations of extinction. The maximum standard deviation of the study obtained for $10^{10}$ realisations is about $8 \%$ : It corresponds to functions associated with rays issued from the third interfacial scattering, the most rare phenomena, i.e. $\left.G_{\text {ext aba }}^{S(3)}, P_{a a b a}^{S(3)}, P_{s c a b a}^{S(3)}\right)$.

\subsection{Results}

In order to remain consistent with the properties of common high temperature insulation felts, the fibres a of diameter $d$ are considered as weakly absorbing and characterised by an absorption optical thickness $\kappa_{a} d=0.3$ and a ratio of refractive indices $n_{a} / n_{b}=2$. In these conditions fibres trap the radiation.
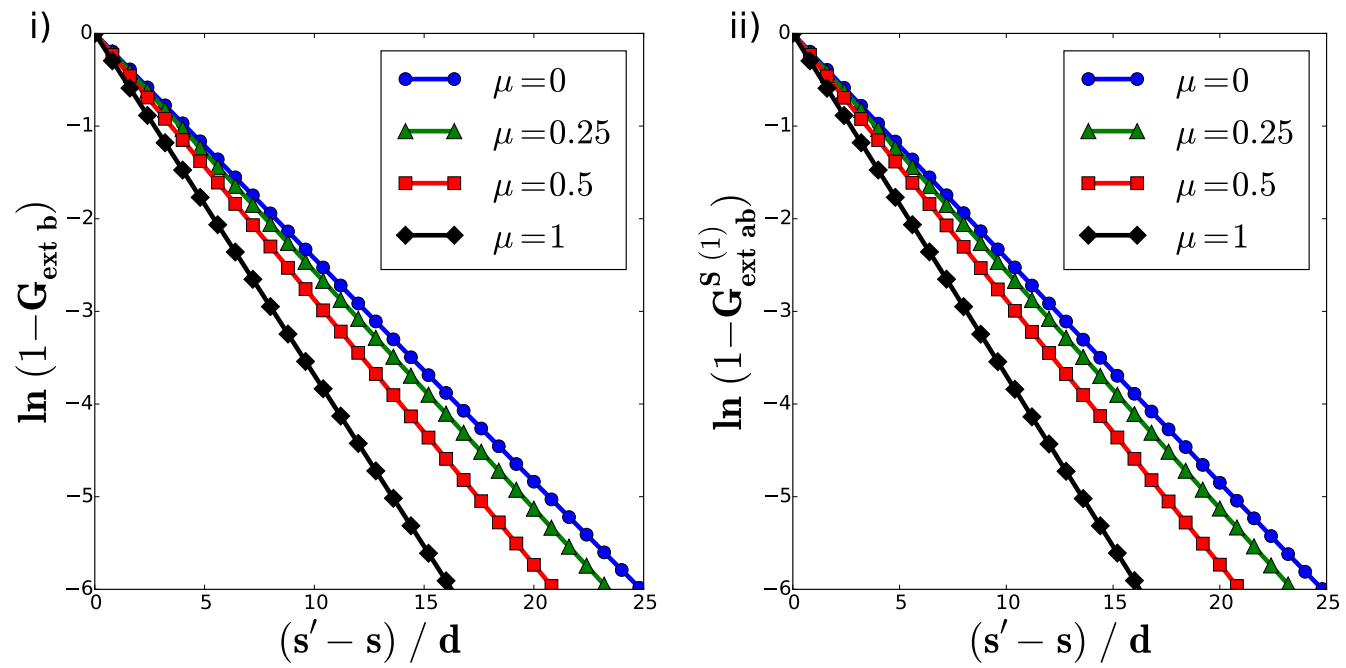

Figure 4: Extinction cumulative distribution functions of the transparent phase $\mathrm{b}$ for different values of $\mu=\cos \theta$ : i) $G_{\text {ext } b}$ associated with isotropic volume source points; ii) $G_{\text {ext } a b}^{S(2)}$ associated with interfacial scattered rays (path 2 of Fig.3).

$G_{\text {ext ab }}^{S(2)}$, the first extinction cumulative distribution function associated with the transparent phase b and corresponding to the path 2 of Fig.3, is compared in Fig.4 with $G_{\text {extb }}$ associated with isotropic volume shots within b. As both results rigorously follow an exponential law for any direction, the homogenised phase $\mathrm{b}$ is Beerian. These results are similar to those pointed out in Ref.[3] in the case of a medium with 
opaque and transparent phases: For a Beerian homogenised phase, extinction cumulative distribution functions issued from isotropic volume source points or from interfacial points are identical. In practice it is easier and more accurate to shoot rays from random isotropic volume source points. Consequently $G_{\text {ext }}\left(\mathbf{u}, s^{\prime}-s\right)$ is used in the following. Extinction by b is then simply characterised by an extinction coefficient $\beta(\mathbf{u})$, obtained for instance at the optically thin limit: $\beta(\mathbf{u})=\frac{\mathrm{d} G_{\text {extb }}}{\mathrm{d}\left(s^{\prime}-s\right)}\left(\mathbf{u}, s^{\prime}-s=0\right)$. As expected the extinction lengths within the homogenised phase $\mathrm{b}$ are maximum in the preferential plane of orientation of the cylinders $(\mu=0)$, and minimum in the directions $\mu=-1$ and $\mu=1$ normal to this plane (see Fig.2). 

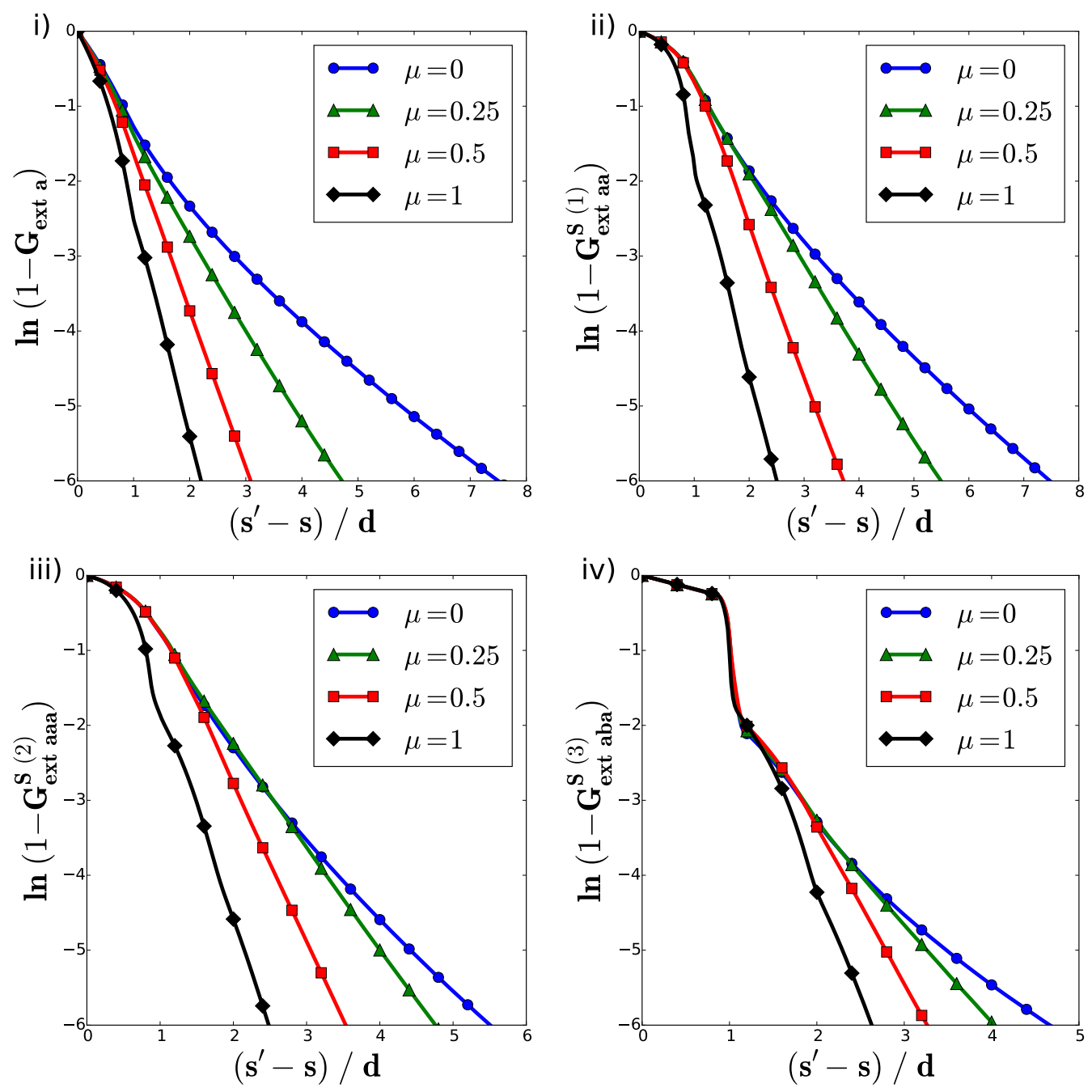

Figure 5: Extinction cumulative distribution functions of phase a (fibres) for different values of $\mu=\cos \theta$. i) $G_{\text {ext a }}$ associated with isotropic volume emission points; ii) $G_{\text {ext aa }}^{S(1)}$; iii) $G_{\text {ext aaa }}^{S(3)}$; iv) $G_{\text {ext aba }}^{S(5)}$ (by using notations of Sec.2.1.1 and Fig.3.)

As shown in Fig.5, the extinction cumulative distribution functions associated with isotropic volume emission within the fibres a and with successive interfacial scattering source terms within a strongly differ and strongly differ from an exponential behaviour: The homogenised phase a is, in general, strongly non Beerian and extinction and scattering coefficients have no more physical meaning. Consequently, the complete original model introduced in Secs.2.1 and 2.2 has to be used. 
From a practical point of view, almost all the emitted power bundles are absorbed after a few scattering events. The extinction cumulative distribution function associated with the Beerian homogenised phase $\mathrm{b}$ is accurately determined. For the non Beerian homogenised phase a, $G_{\text {exta }}, G_{\text {extaa }}^{S(1)}, G_{\text {extaaa }}^{S(3)}, G_{\text {ext aba }}^{S(5)}$ and $G_{\text {ext abba }}^{S(6)}$ are also accurately determined. As only a few bundles are involved, the following extinction cumulative distribution functions, defined in Sec.2.1.1, $G_{\text {extacHaa }}$ and $G_{\text {extacHab }}$ are taken equal to $G_{\text {ext aaa }}^{S(3)}$ and $G_{\text {ext abba }}^{S(6)}$, respectively.

In the core of the medium, generalised extinction and scattering coefficients at equilibrium are defined for the absorbing phase a and the transparent phase $\mathrm{b}$ by equations Eqs.23 and the following development. The transverse optical thicknesses of a fiber associated with these quantities and with the absorption coefficient of the phase a are compared in Fig.6.
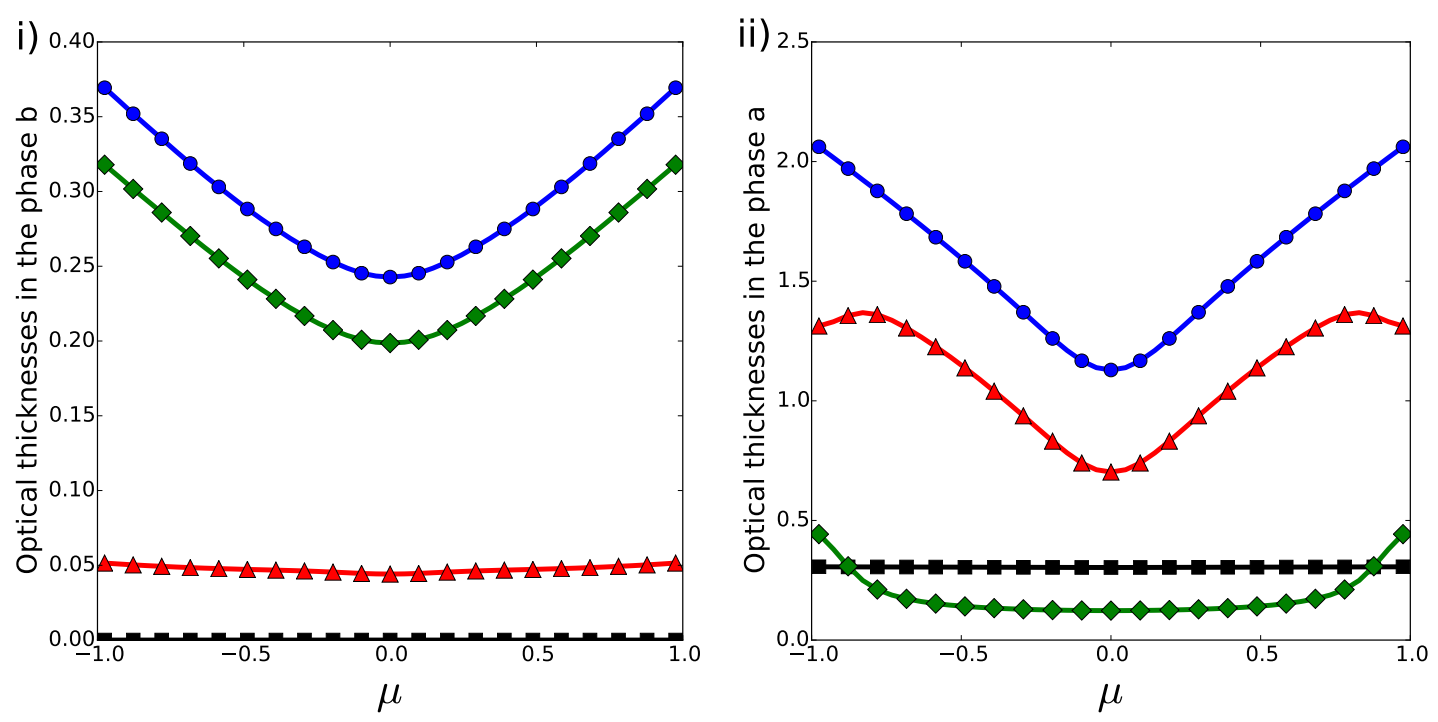

Figure 6: Optical thicknesses based on $\circ$ : Generalised extinction coefficients $\left(B_{c} d\right) ; \triangle$ : Generalised internal scattering coefficients $\left(\Sigma_{c c} d\right) ; \diamond$ : Generalised external scattering coefficients $\left(\Sigma_{c d} d\right)$ and $\square$ : Absorption coefficients $\left(\kappa_{a} d=0.3\right.$ and $\left.\kappa_{b} d=0\right)$;

i)For the transparent phase $b(c=b, d=a)$; ii) For the absorbing phase a (fibres) $(c=a, d=b)$.

Extinction in the transparent phase $\mathrm{b}$ is mainly due to external scattering, but in the absorbing phase a to internal scattering, due to the large ratio of the refractive indices $\left(n_{a} / n_{b}=2\right)$. Internal scattering in phase $\mathrm{b}$ is practically independent of 
$\mu$, as the cylinders practically are orthogonal to the $z$ axis: The local normal unit vector distribution is then practically independent of $\mu$. In phase a (fibres), as $n_{a} / n_{b}=2$, the total reflection phenomenon (for $\mu<0.86$ ) explains the change in slope of the generalised internal scattering coefficient and the weak value of the generalised external scattering coefficient for $|\mu|<0.86$, as shown in Fig.6.b

For $\mu=-1$ and $\mu=1$ the rays are mainly normal to the cylinders, the transmissivity is maximal and decreases with the angle. When the critical angle is reached $\left(\mu_{\text {crit }}=\cos [\arcsin (1 / 2)] \approx 0.86\right)$ the rays are mainly reflected and internal scattering is the predominant mode of extinction. Absorption in the fibre volume is Beerian and isotropic, i.e independent of $\mu$ (Fig.6.b). Finally, a large part of radiation propagating in the transparent phase $\mathrm{b}$ is transmitted towards the phase a (fibres) and then trapped by multiple reflections until absorption occurs. The fibres behave as waveguides.

The phase functions a priori depend on the incidence direction $\mathbf{u}_{1}\left(\mu_{1}, \phi_{1}\right)$ and the scattering one $\mathbf{u}(\mu, \phi)$. Due to the statistical axisymmetry of the generated medium, they are independent of $\phi_{1}$ and depend on $\mu_{1}, \mu$ and $\phi-\phi_{1}$. But as the thermal boundary conditions applied to the medium are also assumed axisymmetric, radiative transfer only occurs along $z$ and the practical phase functions defined in the following only depend on $\mu_{1}$ and $\mu$. Different phase functions are plotted in Fig.7.

For the Beerian transparent homogenised phase b, all phase functions associated with internal scattering within b (upper left and right Figs.7) are identical, whatever the type of source points (of volume or interfacial), as previously seen for a porous medium with a transparent and an opaque phase[3]. It is also the case for all phase functions associated with external scattering from b to a (lower left Fig.7). On the contrary, for the non Beerian homogenised phase a (fibres), the phase functions associated with internal and external scattering issued from different types of source points clearly differ: An example of internal scattering cases is shown in middle Figs.7. 

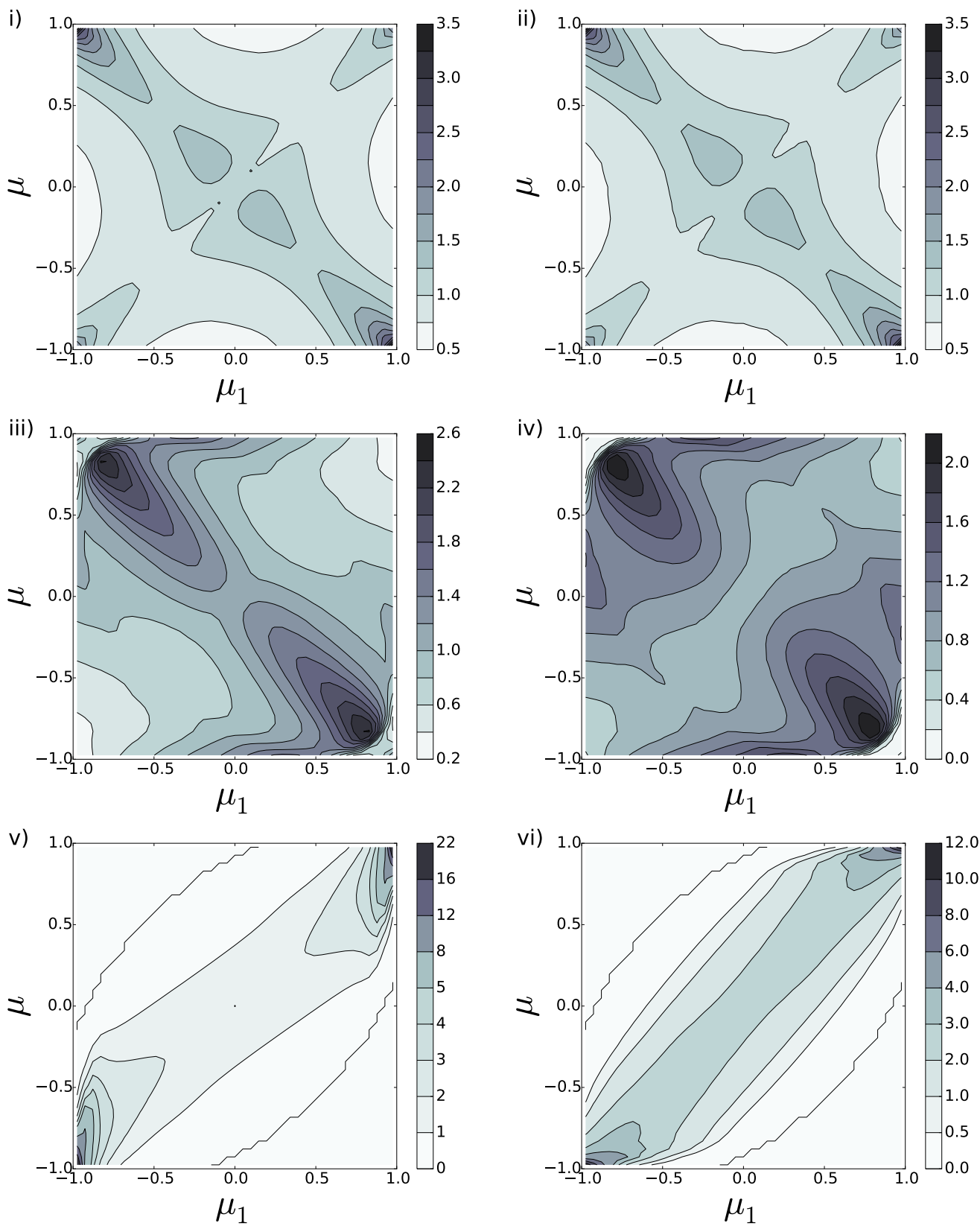

Figure 7: Phase functions. i) $p_{b}\left(\mu_{1}, \mu\right)$, internal scattering within b (Beerian model, random volume source points); ii) $p_{a b b}\left(\mu_{1}, \mu\right)$ internal scattering within b following transmission from a (interfacial source points); iii) $p_{a a}\left(\mu_{1}, \mu\right)$ first internal scattering within a; iv) $p_{a a a}\left(\mu_{1}, \mu\right)$ (second internal scattering within a ; v) $p_{b a}\left(\mu_{1}, \mu\right)$, external scattering from $\mathrm{b}$ to a (Beerian model, random volume source points); vi) $p_{a b}\left(\mu_{1}, \mu\right)$ (first external scattering from a to b). 
As the medium statistically presents both a revolution symmetry axis $z$ and a plane of symmetry perpendicular to the $z$ axis, it also presents a central symmetry. For the directions parallel to the $z$ axis $(\mu=-1$ and $\mu=1)$ the probability of internal scattering within the transparent phase $b$ is maximum in the specular directions, as shown in the upper left Fig.7. It is not the case for the probability of internal scattering within the fibre phase a (see lower Figs.7): This fact is due to the total reflection phenomenon, which occurs for $\mu_{\text {crit }}=0.86$ : Internal scattering is the only extinction phenomenon beyond $\mu_{\text {crit }}$.

The phase function associated with internal scattering within the transparent phase $b$ presents, in addition to the central symmetry, two other symmetries associated with diagonal lines in upper Figs.7, which correspond to the transformations: $\left(\mu_{1}, \mu\right) \rightarrow$ $\left(\mu, \mu_{1}\right)$ and $\left(\mu_{1}, \mu\right) \rightarrow\left(-\mu,-\mu_{1}\right)$. Indeed, the generalised internal scattering coefficient of $\mathrm{b}$ is practically independent of $\mu_{1}$, as shown in left Fig.6, as for a statistically isotropic medium: Consequently the reciprocity theorem (Eq.A.2) leads to the equality between $p_{b b}\left(\mu_{1}, \mu\right)$ and $p_{b b}\left(-\mu,-\mu_{1}\right)$. On the other hand, as the generalised internal scattering coefficient of a is strongly anisotropic, as shown in right Fig.6, the previous symmetries do not appear in the phase function $p_{a a}$.

\section{Application to radiative transfer}

\subsection{Transfer model based on GRTEs}

The homogenised phase a of volume fraction $\Pi_{a}$ and refractive index $n_{a}$ is the only emitting and absorbing phase of the medium. It is characterised by an absorption coefficient $\kappa_{a}$, assumed independent of radiation frequency. As multiple scattering events within a and b occur, many paths possibly couple emission by a in the direction $\mathbf{u}(\mu, \phi)$ from a volume element $\mathrm{d} V_{i}$ of the porous medium around the point $M_{i}\left(\mathbf{r}_{i}\right)$, to absorption by a within a volume element $\mathrm{d} V_{j}$ around the point $M_{j}\left(\mathbf{r}_{j}\right)$. Consequently, the global radiative power $\mathcal{P}_{a i j}^{e a}$ emitted by a in a direction $\mathbf{u}(\mu, \phi)$ from $\mathrm{d} V_{i}$ and absorbed by a within $\mathrm{d} V_{j}$ cannot easily be given by an explicit expression. It will be statistically calculated.

The homogenised phase a is discretised in isothermal volume elements $V_{i}$ of which the temperature is $T_{i}$. $\mathcal{P}_{a}^{e}$, the global power emitted by $V_{i}$, is equal to $4 \Pi_{a} V_{i} \kappa_{a} n_{a}^{2} \sigma T_{i}^{4}$. 
The fraction of this power emitted by $\mathrm{d} V_{i}$ in the elementary solid angle $\mathrm{d} \Omega_{a}(\mathbf{u})$ writes[31, 32]

$$
\frac{\mathrm{d} \mathcal{P}_{a i}^{e}}{\mathcal{P}_{a i}^{e}}=\left(\frac{\mathrm{d} \mathbf{r}_{i}}{V_{i}}\right)\left(\frac{\mathrm{d} \mu}{2}\right)\left(\frac{\mathrm{d} \phi}{2 \pi}\right)=f_{r}\left(\mathbf{r}_{i}\right) \mathrm{d} \mathbf{r}_{i} f_{\mu}(\mu) \mathrm{d} \mu f_{\phi}(\phi) \mathrm{d} \phi,
$$

where $f_{r}\left(\mathbf{r}_{i}\right), f_{\mu}(\mu)$ and $f_{\phi}(\phi)$ are the distribution functions characterising the emission law. The boundaries of the whole porous medium are discretised in isothermal surface elements $S_{j}$ of which the temperature is $T_{j} . \mathcal{P}_{c j}^{e}$, the global power emitted by $S_{j}$, assumed to be a black body, within the phase c (c=a, b) is equal to $S_{j} n_{c}^{2} \sigma T_{j}^{4}$. The fraction of this power emitted by $\mathrm{d} S_{j}$ in the elementary solid angle $\mathrm{d} \Omega_{c}(\mathbf{u})$ writes[31, 32]

$$
\frac{\mathrm{d} \mathcal{P}_{c j}^{e}}{\mathcal{P}_{c j}^{e}}=\left(\frac{\mathrm{d} \mathbf{r}_{j}}{S_{j}}\right)(2 \mu \mathrm{d} \mu)\left(\frac{\mathrm{d} \phi}{2 \pi}\right)=f_{r}^{S}\left(\mathbf{r}_{j}\right) \mathrm{d} \mathbf{r}_{j} f_{\mu}^{S}(\mu) \mathrm{d} \mu f_{\phi}^{S}(\phi) \mathrm{d} \phi,
$$

where $f_{r}^{S}\left(\mathbf{r}_{j}\right), f_{\mu}^{S}(\mu)$ and $f_{\phi}^{S}(\phi)$ are the distribution functions characterising the surface emission law. Emission is then classically modeled by a huge number of shots of bundles $t$ of equal power which, at the statistical limit of large numbers, follow the previous emission statistical laws. Every shot is characterised by discrete values of the quantities ruling emission $\left(\mathbf{r}_{i t}, \mu_{t}, \phi_{t}\right)$ obtained from samplings of corresponding random numbers. The complete path associated with a shot $t$ is defined by successive scattering events within the phases a and $\mathrm{b}$ up to absorption within a volume $V_{j}$ of the phase a or a boundary surface of all the power of the bundle. For a given shot, these events are defined by following the classical forward Monte Carlo method, in which successive extinction distances are also obtained from samplings of random numbers $r$ in the range $[0,1]$, which are values of the associated extinction cumulative distribution functions: An extinction length is then given by: $l_{e x t}=G_{e x t}^{-1}(r)$. Note that, as the medium is statistically homogeneous, the cumulative distribution function of rays issued from boundaries is identical tho this one associated with isotropic volume emission.

Emission is then classically modeled by a huge number of shots of bundles $t$ of equal power which, at the statistical limit of large numbers, follow the previous emis- 
sion statistical laws. Every shot is characterised by discrete values of the quantities ruling emission $\left(\mathbf{r}_{i t}, \mu_{t}, \phi_{t}\right)$ obtained from samplings of corresponding random numbers. The complete path associated with a shot $t$ is defined by successive scattering events within the phases a and b up to absorption within a volume $V_{j}$ of the phase a of all the power of the bundle. For a given shot, these events are defined by following the classical forward Monte Carlo method, in which successive extinction distances are also obtained from samplings of random numbers $r$ in the range $[0,1]$, which are values of the associated extinction cumulative distribution functions: An extinction length is then given by: $l_{\text {ext }}=G_{\text {ext }}^{-1}(r)$.

For every extinction event, the type of extinction is determined, from Eqs. 5 or 6 , by the sampling of another random number. In the case of internal or external scattering within a or $\mathrm{b}$ the calculation is iterated. It is interrupted when extinction is due to absorption: The bundle power is then attributed to the volume cell of the extinction point. In these conditions, the global power emitted by a from $V_{i}$ and absorbed by a within $V_{j}$ writes

$$
\mathcal{P}_{a i j}^{e a}=\mathcal{P}_{a i}^{e} \frac{N_{i j}^{a}}{N_{i}}
$$

where $N_{i}$ is the total number of bundles of same power shot from $V_{i}$ and $N_{i j}^{a}$ the number of these bundles finally absorbed by $V_{j}$.

The reciprocity theorem allows the global radiative power $\mathcal{P}_{a j i}^{e a}$ emitted by a within $\mathrm{d} V_{j}$ and absorbed by a within $\mathrm{d} V_{i}$ to be expressed as

$$
\mathcal{P}_{a j i}^{e a}=\mathcal{P}_{a i j}^{e a} \frac{I^{\circ}\left(T_{a j}\right)}{I^{\circ}\left(T_{a i}\right)},
$$

and the thermal power limited to radiative transfer within the absorbing phase a per unit volume of the whole porous medium then writes at the point $M_{i}$

$$
P_{a i}^{R}=\frac{1}{V_{i}} \sum_{j} \mathcal{P}_{a i j}^{e a}\left(\frac{I^{\circ}\left(T_{a j}\right)}{I^{\circ}\left(T_{a i}\right)}-1\right) .
$$




\subsection{Transfer model based on Fourier's law}

The thermal problem defined in the introduction of Sec.3 is one dimensional along $z$. The thermal power limited to radiative transfer within the absorbing phase a per unit volume of the whole porous medium is simply obtained from the thermal balance of the whole porous medium belonging to the slice between $z$ and $z+\mathrm{d} z$. The only fluxes involved in this balance are the radiative conduction fluxes through the phases $\mathrm{a}$ and $\mathrm{b}$, at the boundaries $z$ and $z+\mathrm{d} z$. An elementary calculation leads to

$$
P_{z}^{F R}=P_{a z}^{F R}=\frac{\partial}{\partial z}\left(k_{a}^{R} \frac{\partial T_{a}}{\partial z}\right)+\frac{\partial}{\partial z}\left(k_{b}^{R} \frac{\partial T_{a}}{\partial z}\right)
$$

where $k_{a}^{R}$ and $k_{b}^{R}$ are the conductivities of the medium along $z$, introduced in Sec.2.4, which depend on the temperature $T_{a}(z)$. As only the phase a emits and absorbs radiation, this power per unit volume only occurs within the phase a. Finally, in this

global approach, it has not been necessary to express the power exchanged between the two phases, as discussed in the introduction of Sec.2.4.

\subsection{Results}

The fibre felt is infinite in the $\mathrm{x}$ and $\mathrm{y}$ directions: Perfect specular reflection laws are applied at the corresponding boundaries $x=0, y=0, x=100 d$ and $y=100 d$. The thickness $D$ of the fibre felt also is $100 d$ in the z direction, divided in 400 cells of equal sizes. It is bounded by two walls at which temperature or flux is imposed.

For an imposed linear temperature field within all the homogenised phase a, the radiative power field within a per unit volume of the whole porous medium $P_{a}^{R}$ given by the coupled GRTEs model (Eq.47) for different transverse optical thicknesses of fibres $\left(\kappa_{a} d=0.3,10\right.$ and 100) is plotted in Fig.8. It is also compared in the same cases with the radiative power field given by the Fourier's model. In the core of the medium, the results associated with the Fourier's law agree with the GRTE's ones only in the case of very high values of $\kappa_{a} d$ (lower Fig.8), i.e when a is an opaque medium. According to the Gomart's criterion (Eq.20), the Fourier's model is not valid when a is semi transparent. When a becomes opaque, this medium can be represented by an effective medium occupying the volume of the phase b, which becomes semi transparent, as commonly done (see Ref.[16] for instance) and the 
Fourier's law can be applied. Moreover, as expected the Fourier's law is never valid near the walls, even for high values of $\kappa_{a} d$, in particular near the hot wall.
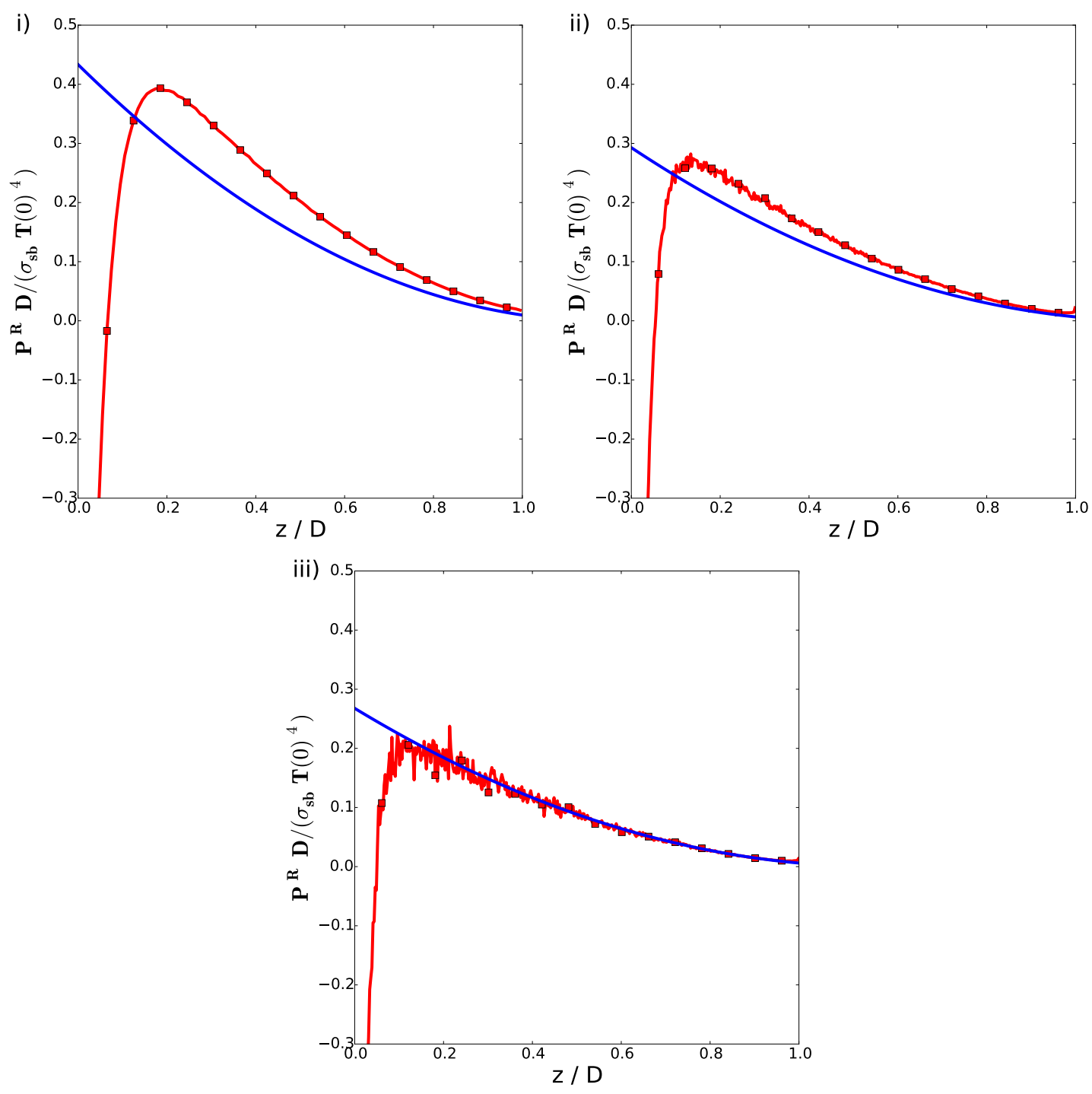

Figure 8: Dimensionless radiative power field for an imposed linear temperature field in the fibre phase; Two boundary black walls at $T_{a}(0)=2000 K$ and $T_{a}(D)=300 K$ : i) $\kappa_{a} d=0.3$; ii) $\kappa_{a} d=10$; iii) $\kappa_{a} d=100 ; \square$ : coupled GRTEs model, - : Fourier's model associated with coupled RTEs.

In the case of temperatures imposed at the two boundaries $z=0$ and $z=D$ of the porous medium $\left(T_{a}(0)=T_{0}\right.$ and $\left.T_{a}(D)=T_{1}\right)$, the temperature field within the phase 
a is iteratively determined in radiative steady state conditions, i.e. by solving $P_{a i}^{R}=0$ for the $N_{c}$ cells $i=1, \ldots, N_{c}$ with these boundary conditions. The results are shown for the realistic case $\kappa_{a} d=0.3$ in Fig.9, where the temperature field issued from the coupled GRTEs is compared with the one issued from the Fourier's model associated with an opaque phase a. Contrary to the previous case, very important errors occur with the Fourier's model near the cold wall, where the logarithmic derivative of the temperature is the most important, which is consistent with the Gomart's criterion of validity of the Fourier's law given by Eq.20. On the other hand, the temperature jump at the boundaries, well predicted by the GRTE's model, is typical of a purely radiative problem (in void for instance). The conductive Fourier's model imposes a non physical continuity of the temperature field in particular at the cold wall.

The Fourier's law overestimates the temperature gradient in the medium. In these conditions, the critical mechanical strength of such a material is underestimated. An accurate computation of the radiative transfer by the coupled GRTE's permits to improve the design.

In the case of an imposed wall flux at $z=0$ and an imposed wall temperature at $z=D$ (Fig.10), the maximum discrepancies between Fourier's and GRTEs results also are located near the cold wall due the same reason as previously. Moreover, the global absolute discrepancy is more important, around $100 \mathrm{~K}$ in the core of the medium. The Fourier's model overestimates the temperature values and consequently underestimates the critical mechanical strength of the material. 

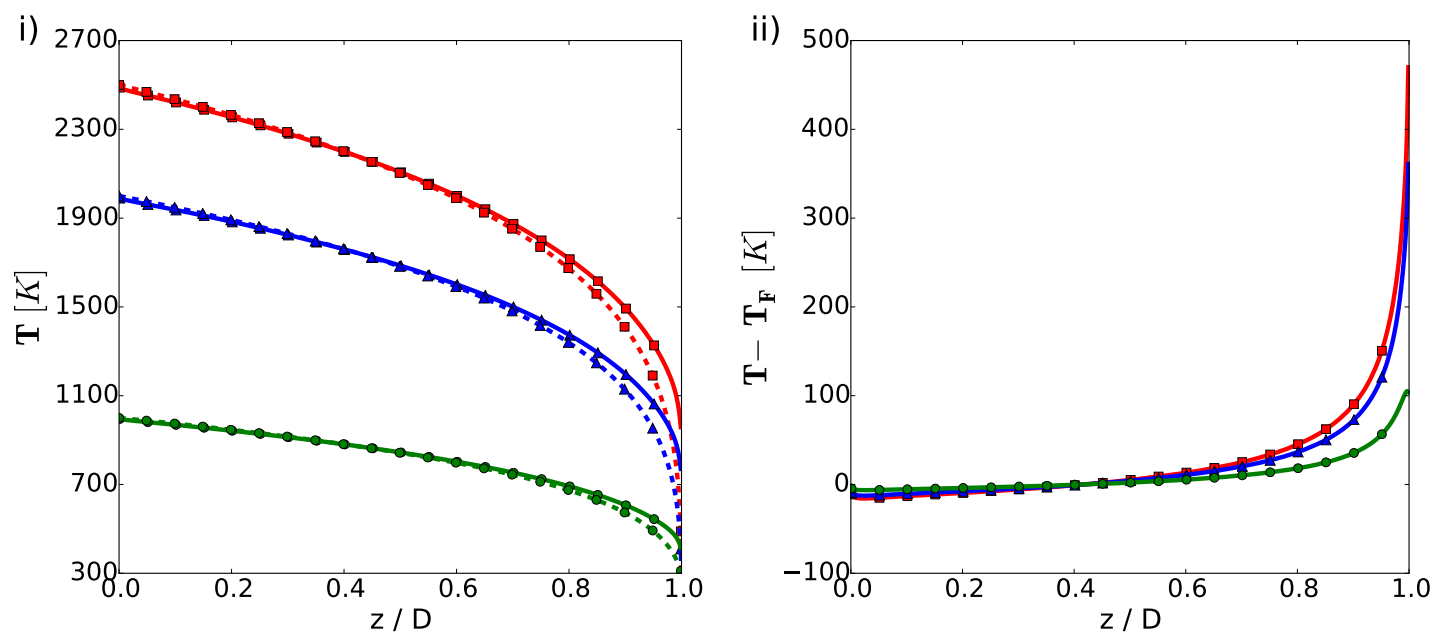

Figure 9: i) Temperature field when the fibrous medium is bounded by two black walls; $\square: T(0)=$ $2500 K, \triangle: T(0)=2000 K, \circ: T(0)=1000 K$; In all cases: $T(D)=300 K$.; (- -) coupled GRTEs model, $(\cdots)$ Fourier's model associated with coupled RTEs; ii) Residuals.
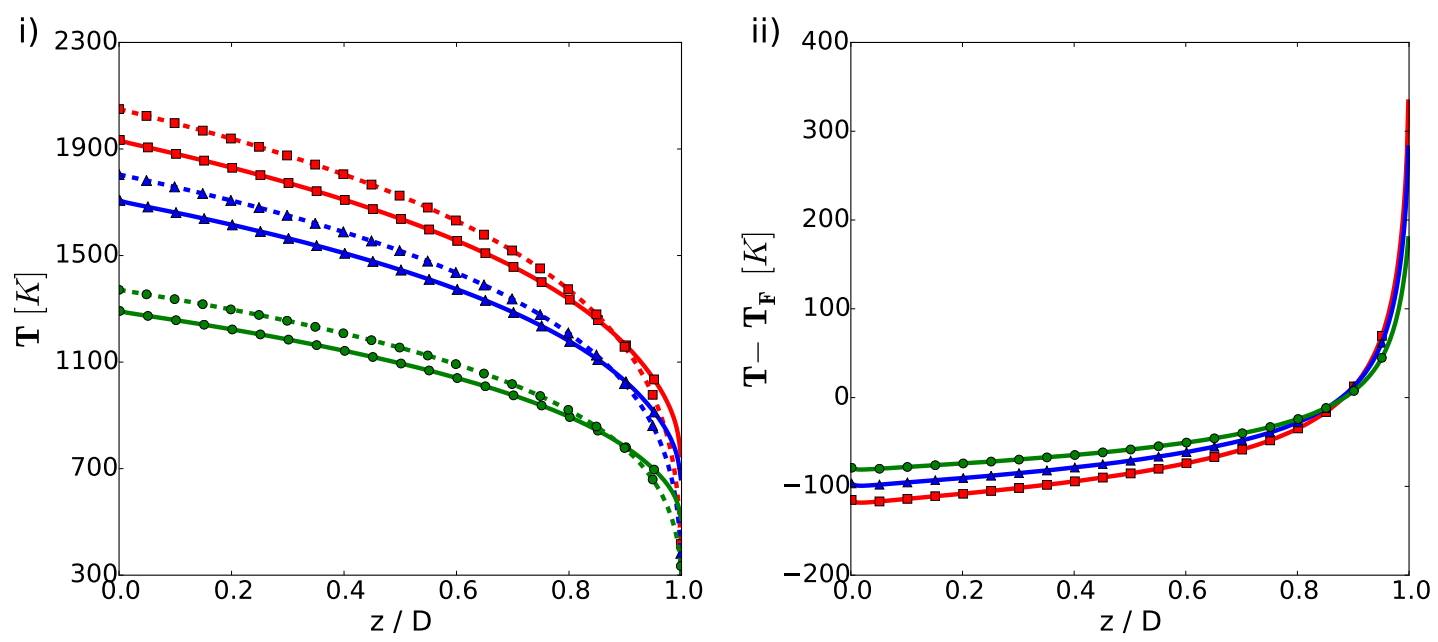

Figure 10: i) Temperature field when a fibrous medium is submitted to an imposed flux at $z=0$ and bounded by a black wall at $z=D ; \square: \varphi(0)=50 \mathrm{kWm}^{-2}, \Delta: \varphi(0)=30 \mathrm{kWm}^{-2}, \circ: \varphi(0)=$ $10 \mathrm{kWm}^{-2} ; T(D)=300 K$; (- -): coupled GRTEs model, $\cdots$ :Fourier's model associated with coupled RTEs; ii) Residuals.

\subsection{Influence of key parameters}

The insulation efficiency of the felt of fibres can be optimised vs three key parameters: The transverse optical thickness of a fibre $\kappa_{a} d$, the ratio of the refractive 
indices $n_{a} / n_{b}$ and the fibre volume fraction $\Pi_{a}$. The two first parameters are input parameters of the model for a given material morphology, and the last one is varied by generating more or less cylinders in the same volume. The test case corresponds to a felt limited by two parallel black walls of imposed temperatures. Note that $\kappa_{a} d$ and $n_{a} / n_{b}$ are independent parameters only when the imaginary part $\chi_{a}$ of the complex optical index of a, which rules absorption by a, is small compared to $n_{a}$ and $n_{b}$. For common alumina and zirconia, characterised by $n_{a} / n_{b} \simeq 2$, the reference value $\kappa_{a} d=0.3$ corresponds to fibres of diameter close to $20 \mu \mathrm{m}$. But the value of $\kappa_{a}$ strongly depends of the impurity amount within the matter. High values of $\kappa_{a} d$, for instance $\kappa_{a} d=100$, can not be obtained obtained for fibres of sub- $m m$ diameter.

The aim of this Section is to minimise the flux through the system, calculated from the coupled GRTEs. The results, plotted in Fig.11, are limited to a pure radiative transfer, i.e. when conduction transfer in the two phases can be neglected. As seen in upper left Fig.11, the radiative flux is a decreasing function of $\kappa_{a} d$, which could be expected, and the radiative flux is divided by a factor approximately 2 when $\kappa_{a} d$ varies from 0.1 to 100 .

A strong flux appears in Fig.11 ii) for the particular case $n_{a}=n_{b}$ because the interfaces then do not exist for the radiation field: Only absorption in fibres plays a role in radiative transfer. When $n_{a}<n_{b}$ (fibres embedded in a particular transparent resin) total reflection occurs in the phase b: The rays are trapped in this phase and the flux vanishes. The same comment can be made when $n_{a}>n_{b}$ : The rays are then trapped in the phase a and this trapping effect increases with the refractive index ratio.

It is easily conceived that the radiative flux exhibits a local maximum for $n_{a} / n_{b}=$ 1. Moreover, starting from a value of $n_{a} / n_{b}$ sufficiently greater than 1 and letting this quantity grow up to infinity, the interfacial Fresnel's reflectivity tends to the value of 1 , and this facilitates the radiative transfer as compared to a situation where at an interface the incident radiation is split into a reflected part and a transmitted part. On the basis of these arguments, a local minimum of the radiative flux vs the ratio $n_{a} / n_{b}$ is expected at a value of $n_{a} / n_{b}$ greater than 1 . The upper right Fig.11 shows that this minimum occurs when the ratio $n_{a} / n_{b}$ is in the range $[2,4]$. Alumina and 
zirconia present this last property when $\mathrm{b}$ is the vacuum or a gas.
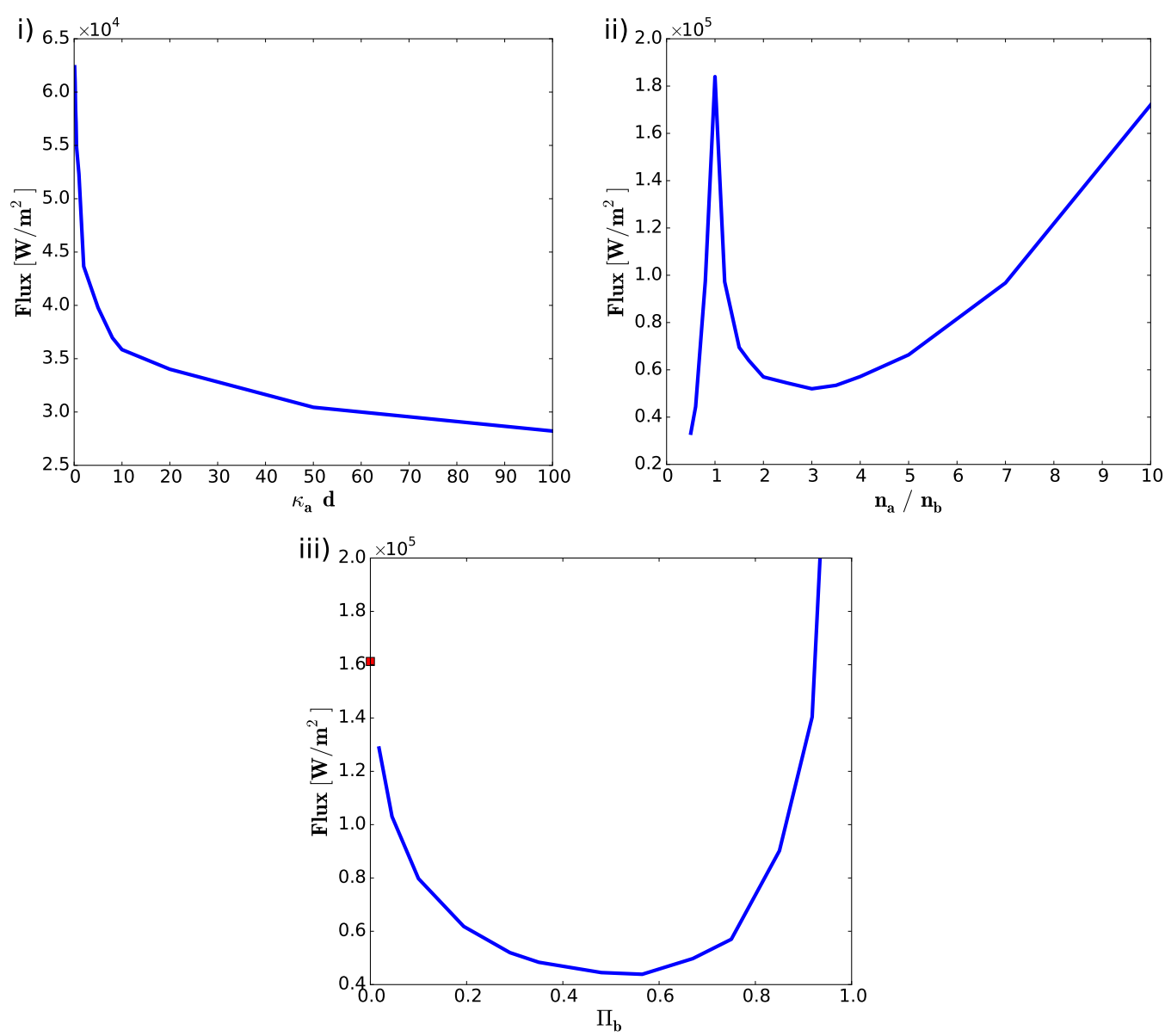

Figure 11: Radiative flux through the fibrous medium: i) vs absorption optical thickness; ii) vs optical index ratio; iii) vs porosity; The medium is bounded by two black walls at $T(0)=2000 \mathrm{~K}$ and $T(D)=300 K$; Other parameter values are: $\kappa_{a} d=0.3, n_{a} / n_{b}=2, \Pi_{b}=0.75$.

In the lower Fig.11 (radiative flux vs the porosity $\Pi_{b}$ ), the two extreme values of this curve are easily analysed. When $\Pi_{b}=1$, there are no more fibres in the material, and as the phase $\mathrm{b}$ is assumed transparent, the radiative flux tends to infinity. When $\Pi_{b}=0$, the material is homogeneous, absorbing (of absorption coefficient $\kappa_{a}$ ) and non scattering, and within the frame of the radiative conductivity model, the flux writes $4 n_{a}^{2} \sigma_{s b}\left[T^{4}(0)-T^{4}(L)\right] /\left(3 \kappa_{a} D\right)$ with $\sigma_{s b}$ the Stefan-Boltzmann constant, which leads to a quite high value. Between the extreme values associated with $\Pi_{b}=0$ and 
$\Pi_{b}=1$, the radiative flux profile shown in lower Fig.11 exhibits a minimum value, which may be interpreted as follows: Quite logically, the more fibres are present within the material, the more absorption is brought to it, and consequently the lower the radiative flux. But it is also conceived that the more fibres are present within the material, the more rare the scattering events at the fibre boundaries, and consequently the higher the radiative flux. The superposition of these two antagonist effects leads to a minimum value of the flux for a value of the porosity $\Pi_{b}$ in the range $[0.4,0.6]$.

Such a study has to be generalised by accounting for conduction within the two phases.

\section{Conclusion}

An original statistical model of characterisation of the radiative properties of the a priori non Beerian homogenised phases of a strongly anisotropic porous medium, with a semi transparent phase and a transparent one (STT), has first been developed. The general case of reflection and transmission laws at interfacial scale has been treated. This model is based on all the extinction cumulative distribution functions and scattering (or absorption) cumulative probabilities associated with the successive sets of internal and external scattering events, by generalising the approach of Ref.[3]. Moreover, all the associated general phase functions, depending on both the incidence and scattering directions, have been for the first time ab initio determined for a STT medium.

An original statistical model of radiative transfer based on coupled Generalised Radiative Transfer Equations (GRTEs) associated with the two homogenised phases has also been developed. The coupling terms are due to the external scattering terms, associated with transmission at interfacial scale. It has been shown that a radiative Fourier's model, based on radiative conductivity tensors, is not valid for the STT case, if the particular case for which the semi transparent phase becomes opaque and the trivial case of a quasi isothermal medium are excepted.

The two models have been applied to a fibrous medium (fibre felt) for thermal insulation at high temperature: A set of overlapping finite absorbing cylinders within 
a transparent matrix. It has been shown that the fibrous phase is strongly non Beerian, and that the transparent one is Beerian, due to particular symmetries associated with cylinders.

Finally a parametric study of the insulation properties of the medium has been carried out by only accounting for radiation transfer for optimisation of the design. Such a study has to be generalised by accounting for conduction within the two phases.

\section{Appendix A. Some properties of the scattering source terms}

The case of external scattering from c towards $d$ is only considered. The results associated with internal scattering are deduced by taking $\mathrm{c}=\mathrm{d}$. External scattering from a Beerian medium $c$ of refractive index $n_{\nu c}$ towards a Beerian medium $d$ of refractive index $n_{\nu d}$ is characterised by a scattering coefficient $\sigma_{\nu c d}(\mathbf{u})$ and a phase function $p_{\nu c d}$. The corresponding scattering source term expressed in medium d per unit volume in the elementary solid angle $\mathrm{d} \Omega_{d}(\mathbf{u})$ then writes

$$
S_{s c \nu c d}(\mathbf{u}) \mathrm{d} \Omega_{d}(\mathbf{u})=\mathrm{d} \Omega_{d}(\mathbf{u}) \int_{4 \pi} \sigma_{\nu c d}\left(\mathbf{u}_{\mathbf{1}}\right) \frac{p_{\nu c d}\left(\mathbf{u}_{1}, \mathbf{u}\right)}{4 \pi} I_{\nu c}\left(\mathbf{u}_{1}\right) \mathrm{d} \Omega_{1 c}\left(\mathbf{u}_{1}\right),
$$

where the phase function $p_{\nu c d}$ associates a scattered intensity, expressed in medium $\mathrm{d}$, with an elementary incident flux characterised by the intensity $I_{\nu c}$ expressed in medium $\mathrm{c}$.

In Ideal Thermal Equilibrium (ITE) conditions, the reciprocity theorem states that the power scattered from the direction $\mathbf{u}_{1}$ of medium c to the direction $\mathbf{u}$ of medium $\mathrm{d}$ is equal to the power scattered from $-\mathbf{u}$ of $\mathrm{d}$ to $-\mathbf{u}_{1}$ of $\mathrm{c}$, i.e.

$$
\begin{gathered}
\sigma_{\nu c d}\left(\mathbf{u}_{1}\right) \Pi_{c} n_{\nu c}^{2} I_{\nu}^{\circ}(T) \mathrm{d} \Omega_{1 c}\left(\mathbf{u}_{1}\right) \frac{p_{\nu c d}\left(\mathbf{u}_{1}, \mathbf{u}\right)}{4 \pi} \mathrm{d} \Omega_{d}(\mathbf{u}) \\
=\sigma_{\nu d c}(-\mathbf{u}) \Pi_{d} n_{\nu d}^{2} I_{\nu}^{\circ}(T) \mathrm{d} \Omega_{d}(-\mathbf{u}) \frac{p_{\nu d c}\left(-\mathbf{u},-\mathbf{u}_{1}\right)}{4 \pi} \mathrm{d} \Omega_{1 c}\left(-\mathbf{u}_{1}\right) .
\end{gathered}
$$

The Clausius theorem writes

$$
n_{\nu c}^{2} \mathrm{~d} \Omega_{1 c}\left(\mathbf{u}_{1}\right)=n_{\nu d}^{2} \mathrm{~d} \Omega_{d}(\mathbf{u})=\mathrm{d} \Omega
$$

where $\mathrm{d} \Omega$, $\mathrm{d} \Omega_{1 c}\left(\mathbf{u}_{1}\right)$ and $\mathrm{d} \Omega_{d}(\mathbf{u})$ are the conjugated elementary solid angles in vac- 
uum, in medium c and medium d, respectively. Moreover,

$$
\mathrm{d} \Omega_{1 c}(\mathbf{u})=\mathrm{d} \Omega_{1 c}(-\mathbf{u}), \quad \mathrm{d} \Omega_{d}(\mathbf{u})=\mathrm{d} \Omega_{d}(-\mathbf{u}), \quad \mathrm{d} \Omega(\mathbf{u})=\mathrm{d} \Omega(-\mathbf{u}) .
$$

In these conditions Equation A.2 becomes

$$
\sigma_{\nu c d}\left(\mathbf{u}_{1}\right) \Pi_{c} \frac{p_{\nu c d}\left(\mathbf{u}_{1}, \mathbf{u}\right)}{4 \pi n_{\nu d}^{2}}=\sigma_{\nu d c}(-\mathbf{u}) \Pi_{d} \frac{p_{\nu d c}\left(-\mathbf{u},-\mathbf{u}_{1}\right)}{4 \pi n_{\nu c}^{2}}
$$

and consequently Equation A.1 now writes, by using Eq.A.5,

$$
S_{s c \nu c d}(\mathbf{u})=\left(\frac{n_{\nu d}^{2} \Pi_{d}}{n_{\nu c}^{2} \Pi_{c}}\right) \sigma_{\nu d c}(-\mathbf{u}) \int_{4 \pi} \frac{p_{\nu d c}\left(-\mathbf{u},-\mathbf{u}_{1}\right)}{4 \pi} I_{\nu c}\left(\mathbf{u}_{1}\right) \mathrm{d} \Omega_{1 c}\left(-\mathbf{u}_{1}\right) .
$$

The phase function $p_{\nu d c}$, for instance, is normalised by

$$
\int_{4 \pi} \frac{p_{\nu d c}\left(-\mathbf{u},-\mathbf{u}_{1}\right)}{4 \pi} \mathrm{d} \Omega_{1 c}\left(-\mathbf{u}_{1}\right)=1
$$

In ITE conditions, the global scattering source term from $\mathrm{c}$ to $\mathrm{d}$ in direction $\mathbf{u}$ expressed in medium $\mathrm{d}$, is then equal to

$$
S_{s c \nu c d}^{\circ}(\mathbf{u})=\sigma_{\nu d c}(-\mathbf{u}) \Pi_{d} n_{\nu d}^{2} I_{\nu}^{\circ}(T)
$$

According to the detailed balance principle this quantity is equal in ITE conditions to the extinction term of the medium $\mathrm{d}$ in the direction $\mathbf{u}$ associated with scattering towards c, i.e. $\sigma_{\nu d c}(\mathbf{u}) \Pi_{d} n_{\nu d}^{2} I_{\nu}^{\circ}(T)$, which leads to

$$
\sigma_{\nu d c}(-\mathbf{u})=\sigma_{\nu d c}(\mathbf{u})
$$

The reciprocity theorem applied in ITE conditions to internal scattering within c also leads to

$$
S_{s c \nu c c}(\mathbf{u})=\sigma_{\nu c c}(-\mathbf{u}) \int_{4 \pi} \frac{p_{\nu c c}\left(-\mathbf{u},-\mathbf{u}_{1}\right)}{4 \pi} I_{\nu c}\left(\mathbf{u}_{1}\right) \mathrm{d} \Omega_{1 c}\left(-\mathbf{u}_{1}\right),
$$

and

$$
\sigma_{\nu c c}(-\mathbf{u})=\sigma_{\nu c c}(\mathbf{u})
$$


[1] D. Baillis and J. F. Sacadura. Thermal radiation properties of dispersed media: theoretical prediction and experimental characterization. Journal of Quantitative Spectroscopy and Radiative Transfer, 67(5):327-363, 2000.

[2] J. Taine and E. Iacona. Upscaling statistical methodology for radiative transfer in porous media: New trends. Journal of Heat Transfer, 134, 2012.

[3] Y. Dauvois, M. Zarrouati, F. Enguehard, J. Taine, and D. Rochais. Comments over homogenisation scales for interfacial emission and scattering by a divided medium: Beerian and non beerian behaviours. International Journal of Heat and Mass Transfer, submitted 2016.

[4] J.L. Consalvi, B. Porterie, and J.C. Loraud. A formal averaging procedure for radiation heat transfer in particulate media. International Journal of Heat and Mass Transfer, 45:2755-2768, 2002.

[5] W. Lipinski, J. Petrasch, and S. Haussener. Application of the spatial averaging theorem to radiative heat transfer in two-phase media. Journal of Quantitative Spectroscopy and Radiative Transfer, 11:253-258, 2010.

[6] M. Quintard and S. Whitaker. Transport in ordered and disordered porous media I: The cellular average and the use of weighting functions. Transport in Ordered and Disordered Porous Media I: The Cellular Average and the Use of Weighting Functions, 14:179-206, 1994.

[7] M. Quintard and S. Whitaker. Transport in ordered and disordered porous media II: Generalized volume averaging. Transport in Porous Media, 1994.

[8] M. Tancrez and J. Taine. Direct identification of absorption and scattering coefficients and phase function of a porous medium by a Monte Carlo technique. International Journal of Heat and Mass Transfer, 47:373-383, 2004.

[9] B. Zeghondy, E. Iacona, and J. Taine. Determination of the anisotropic radiative properties of a porous material by radiative distribution function identification (RDFI). International Journal of Heat and Mass Transfer, 49:2810-2819, 2006. 
[10] J. Petrasch, P. Wyss, and A. Steinfeld. Tomography-based Monte Carlo determination of radiative properties of reticulated porous ceramics. Journal of Quantitative Spectroscopy and Radiative Transfer, 105:180-197, 2007.

[11] F. Bellet, E. Chalopin, F. Fichot, E. Iacona, and J. Taine. RDFI determination of anisotropic and scattering dependent radiative conductivity tensors in porous media: Application to rod bundles. International Journal of Heat and Mass Transfer, 52:1544-1551, 2009.

[12] S. Haussener, P. Coray, W. Lipinski, P. Wyss, and A. Steinfeld. Tomographybased heat and mass transfer characterization of reticulate porous ceramics for high-temperature processing. J. of Heat Transfer, 132(023305):1-9, 2010.

[13] S. Haussener, W. Lipinski, P. Wyss, and A. Steinfeld. Tomography-based analysis of radiative transfer in reacting packed beds undergoing a solid-gas thermochemical transformation. J. of Heat Transfer, 132(061201):1-7, 2010.

[14] M. Chahlafi, F. Bellet, F. Fichot, and J. Taine. Radiative transfer within non Beerian porous media with semitransparent and opaque phases in non equilibrium: Application to reflooding of a nuclear reactor. Int. J. Heat Mass Transfer, 55(13-14):3666-3676, 2012.

[15] S. Haussener, W. Lipinski, J. Petrasch, P. Wyss, and A. Steinfeld. Tomographic Characterization of a Semitransparent-Particle Packed Bed and Determination of its Thermal Radiative Properties. J. Heat Transfer, 131(072701):1-7, 2009.

[16] J. Taine, F. Bellet, V. Leroy, and E. Iacona. Generalized radiative transfer equation for porous medium upscaling: Application to the radiative Fourier law. Int. J. Heat Mass Transfer, 53(19-20):4071-4081, 2010.

[17] M. Zarrouati, F. Enguehard, and J Taine. Radiative transfer within strongly non homogeneous porous media: Application to a slab of packed particles. International Journal of Heat and Mass Transfer, 91:936-947, 2015. 
[18] T.W. Tong and C.L. Tien. Analytical models for thermal radiation in fibrous insulations. Journal of Building Physics, 4:27-44, 1980.

[19] S.C. Lee. Radiative transfer through a fibrous medium: Allowance for fiber orientation. Journal of Quantitative Spectroscopy and Radiative Transfer, 36(3):253-263, 1986.

[20] P. Boulet, G. Jeandel, and G. Morlot. Model of radiative transfer in fibrous media. International Journal of Heat and Mass Transfer, 38(18):4287-4297, 1993.

[21] A. Milandri, F. Asllanaj, G. Jeandel, and J.R. Roche. Heat transfer by radiation and conduction in fibrous media without axial symmetry. Journal of Quantitative Spectroscopy and Radiative Transfer, 74:585-603, 2002.

[22] F. Asllanaj, G. Jeandel, J.R. Roche, and D. Lacroix. Transient combined radiation and conduction heat transfer in fibrous media with temperature and flux boundary conditions. International Journal of Thermal Sciences, 43, 2004.

[23] H.T. Kamdem Tagne and D. Baillis. Radiative heat transfer using isotropic scaling approximation: Application to fibrous medium. Journal of Heat Transfer, 127:1115-1123, 2005.

[24] H.T. Kamdem Tagne and D. Baillis. Reduced models for radiative heat transfer analysis through anisotropic fibrous medium. Journal of Heat Transfer, 132:1-8, 2010 .

[25] S.C. Lee. Scattering phase function for fibrous media. International Journal of Heat and Mass Transfer, 33(10):2183-2190, 1989.

[26] P. Boulet, A. Collin, and J.L. Consalvi. On the finite volume method and the discrete ordinates method regarding radiative heat transfer in acute forward anisotropic scattering media. Journal of Quantitative Spectroscopy $\&$ Radiative Transfer, 104:460-473, 2007. 
[27] M.I. Mishchenko, J.M. Dlugach, E.G. Yanovitskij, and N.T. Zakharova. Bidirectional reflectance of flat, optically thick particulate layers: an efficient radiative transfer solution and applications to snow and soil surfaces. Journal of Quantitative Spectroscopy \&3 Radiative Transfer, 63:409-432, 1999.

[28] B. Hunter and Z. Guo. Applicability of phase-function normalization techniques for radiation transfer computation. Numerical Heat Transfer, 67(Part B):1-24, 2015.

[29] B. Zeghondy, E. Iacona, and J. Taine. Experimental validation of RDFI method predictions of statistically anisotropic porous medium radiative properties. International Journal of Heat and Mass Transfer, 49:3701-3707, 2006.

[30] H. Gomart and J. Taine. Validity criterion of the radiative Fourier law for an absorbing and scattering medium. Physical Review E, 83(2):1-8, 2011.

[31] J. Taine, F. Enguehard, and E. Iacona. Transferts Thermiques. Dunod, Paris, $5^{\text {th }}$ edition, 2014.

[32] L. Tesse, F. Dupoirieux, and J. Taine. Monte carlo modeling of radiative transfer in a turbulent sooty flame. International Journal of Heat and Mass Transfer, 47:555-572, 2004. 